\title{
A Variational Approach for Retrieving Raindrop Size Distribution from Polarimetric Radar Measurements in the Presence of Attenuation
}

\author{
Qing Cao ${ }^{1,2}$, Guifu Zhang ${ }^{1,3}$, and Ming Xue $e^{3,4}$ \\ ${ }^{1}$ Advanced Radar Research Center, University of Oklahoma, Norman, OK 73072 \\ ${ }^{2}$ Hydrometeorology and Remote Sensing Laboratory, University of Oklahoma, Norman, \\ OK 73072 \\ ${ }^{3}$ School of Meteorology, University of Oklahoma, Norman, OK 73072 \\ ${ }^{4}$ Center for Analysis and Prediction of Storms, University of Oklahoma, Norman, OK \\ 73072
}

Accepted by JAMC

July 2012

Corresponding author address:

Dr. Qing Cao

Advanced Radar Research Center \&

Hydrometeorology and Remote Sensing Laboratory

University of Oklahoma

120 David L. Boren Blvd, Suite 4638

Norman OK 73072, USA

E-mail: qingcao@ou.edu

Tel: $(+1) 405-325-0505$

\begin{abstract}
This study presents a two-dimensional variational approach to retrieving raindrop size distributions (DSDs) from polarimetric radar data in the presence of attenuation. A two-parameter DSD model, the constrained-gamma model, is used to represent rain DSDs. Three polarimetric radar measurements, reflectivity $\left(\mathrm{Z}_{\mathrm{H}}\right)$, differential reflectivity $\left(Z_{\mathrm{DR}}\right)$ and specific differential phase $\left(\mathrm{K}_{\mathrm{DP}}\right)$, are optimally used to correct for the attenuation and retrieve DSDs by taking into account measurement error effects. Retrieval results with simulated data demonstrate that the proposed algorithm performs well. Applications to real data collected by the X-band CASA (Center for Collaborative Adaptive Sensing of the Atmosphere) radars and the C-band OU-PRIME (University of Oklahoma - Polarimetric Radar for Innovations in Meteorology and Engineering) radar also demonstrate the efficacy of this approach.
\end{abstract}




\section{Introduction}

The weather radar, due to its high temporal $(\sim 5$ minutes $)$ and spatial $(\sim 1 \mathrm{~km})$ resolutions, is an effective instrument for weather surveillance. In the past decades, much effort has been put into quantitative precipitation estimation (QPE) using radar observations (Doviak and Zrnić, 1993; Bringi and Chandrasekar, 2001). In the early years, rainfall estimation mainly depended on empirical relations, i.e., the radarreflectivity $(Z)$ and rainfall rate $(R) Z-R$ relations. However, it has been realized that Z-R relation has a large variability for different rain types, seasons, locations, etc. Hundreds of Z-R relations have been reported in the literature (Doviak and Zrnic, 1993; Rosenfeld and Ulbrich, 2003), implying that an empirical Z-R relation is limited in representing raindrop size distribution (DSD), which contains the microphysical information of rainfall. The latest polarimetric radar technology has greatly contributed to the improvement of QPE (Bringi and Chandrasekar 2001; Matrosov et al. 2002; Ryzhkov et al. 2005). With additional polarimetric observations, including differential reflectivity $\left(\mathrm{Z}_{\mathrm{DR}}\right)$ and specific differential phase $\left(\mathrm{K}_{\mathrm{DP}}\right)$, DSDs can be better characterized and rain estimation is consequently more accurate. Since accounting for DSD variability is essential for accurate rain estimation, how to model and retrieve DSD well becomes an important research topic. It has been demonstrated that the constraint-gamma (C-G) DSD model (Zhang et al. 2001; Brandes et al. 2002; Cao et al. 2008, 2010), which is more flexible than the most commonly used Marshall-Palmer and exponential DSD models, is suitable for the direct DSD retrieval from two polarimetric radar variables. In this paper, DSD retrieval applying the $\mathrm{C}-\mathrm{G}$ model and polarimetric radar measurements is further studied. Two issues regarding the retrieval are emphasized: i) attenuation correction, and ii) optimal use of radar measurements.

Depending on the radar frequency and the intensity of precipitation, measured radar reflectivity can be greatly attenuated by the precipitation. Shorter radar wavelengths (C-band at $\sim 5 \mathrm{~cm}$ wavelength and $\mathrm{X}$-band at $\sim 3 \mathrm{~cm}$ wavelength) result in stronger attenuation. Without correcting for attenuation, QPE could be heavily underestimated in such situations. Many national weather radar networks in the world (e.g., most European radar networks and part of Chinese operational radar network) operate at $\mathrm{C}$ band. In the United States, an X-band radar network has been suggested (McLaughlin 2010) to complement or replace the current NEXRAD (Next-Generation Radar) network that operates at S-band ( $\sim 10 \mathrm{~cm}$ wavelength). The X-band IP1 (Integrated Project 1) radar network of CASA (Center for Collaborative Adaptive Sensing of the Atmosphere) is a testbed for such a purpose. The attenuation for the X-band IP1 radar is a significant issue for radar-based QPE and microphysical retrieval.

Some methods of correcting for precipitation attenuation can be found in previous studies. For a single-polarization radar, the Hitschfeld-Bordan (H-B) method and its variants can be applied (Delrieu et al. 2000; Zhang et al. 2004; Berne and Uijlenhoet 2006). The deterministic power law relation between the attenuation factor and the radar reflectivity is the basis for $\mathrm{H}-\mathrm{B}$ method and its variants. For dual-polarization radars, the propagation phase (differential phase or specific differential phase) is usually used in attenuation correction algorithms. Those algorithms include the direct phase correction method (Bringi et al. 1990), data fitting method (Ryzhkov and Zrnic 1995), ZPHI algorithm (Testud et al. 2000), self-consistency (SC) method (Bringi et al. 2001) and 
revised SC methods (Park et al. 2005; Vulpiani et al. 2005; Gorgucci and Baldini 2007; Liu et al. 2006; Ryzhkov et al. 2007). The power law relations between the attenuation factor and the specific differential phase are essential for these dual-polarization radar algorithms. Although empirical relations associated with attenuation facilitate the attenuation correction, strong constraints introduced by these relations may sacrifice much of the physical variability of precipitation. An alternate approach is to estimate the attenuation through retrieving precipitation's microphysical information, e.g., DSD (Meneghini and Liao 2007). If the DSD can be well estimated with its variability preserved, the attenuation will consequently be well corrected.

Another issue addressed in this study is the optimal use of multi-frequency radar measurements. Previous QPE or DSD retrievals have mainly applied a deterministic approach; the radar observations are related directly to the estimation without accounting for their error effect. Examples can be seen with empirical QPEs (e.g., Ryzhkov et al. 2005) or direct retrievals (e.g., Zhang et al. 2001; Cao et al. 2008). The useful information from multiple polarimetric measurements is not optimally utilized. Measurement errors, which are different for various radar parameters, may negatively affect attenuation correction and consequent QPE. It is worth noting that the variational method as well as other methods based on optimal estimation theory provides an effective way to account for the error effect and integrate multiple measurements of radar (Hogan 2007; Xue et al. 2009). It is reasonable to expect that the variational method would improve the application of radar data for QPE.

This paper proposes a rain estimation scheme based on polarimetric radar data, which integrates three components (DSD retrieval, attenuation correction and the variational method) as a whole and enables rain estimation to benefit from all of them. More specifically, the proposed scheme is a two-dimensional variational scheme for DSD retrieval. Polarimetric radar data used in this scheme are radar reflectivity of horizontal polarization $\left(\mathrm{Z}_{\mathrm{H}}\right), \mathrm{Z}_{\mathrm{DR}}$, and $\mathrm{K}_{\mathrm{DP}}$ of a plan position indicator (PPI) sweep. The C-G DSD model is used due to its flexibility in representing DSD variability. The state vectors to be variationally retrieved consist of two C-G parameters at every grid point in the analysis region. Attenuation is included in the forward observation operator, and attenuation correction and DSD estimation are accomplished adaptively in the iterative optimization process. Although this scheme is also applicable for the case of multiple station/frequency radars, this study focuses only on the application of monopole radar. The rest of this paper is organized as follows. The methodology of the proposed scheme is detailed in Section 2. The validity of this scheme is demonstrated using simulated Xband radar data in Section 3. Section 4 evaluates the sensitivity of this scheme using the simulated data as well. Real data from X-band IP1 radar and C-band OU-PIRME radar are used for testing in Section 5. The last section provides a summary and some further discussion on the proposed scheme.

\section{Methodology}

\section{a. The variational formulation}

This variational retrieval scheme applies precipitation-attenuated polarimetric radar data $(\mathrm{PRD}), \mathrm{Z}_{\mathrm{H}}^{\prime}, \mathrm{Z}_{\mathrm{DR}}^{\prime}$, and $\mathrm{K}_{\mathrm{DP}}$. The superscript “"” indicates radar measurements with attenuation versus the intrinsic values without attenuation. Gaseous attenuation in

radar measurements is ignored in this study. Theoretically, the application of variational 
scheme requires that the measurement errors have Gaussian distribution (Kalnay, 2002). Uncertainty related to the error model will be introduced in the variational scheme if nonGaussian error exists for observations. The modification of observational cost function may help to mitigate such uncertainty (Koizumi et al. 2005). The error probability density function (PDF) of reflectivity (linear unit) approaches Gaussian when there are sufficient independent samples (Doviak and Zrnic, 1993, section 4.3 and 6.3) and its standard deviation is proportional to the expected value. Xue et al. (2007) investigated the error modeling of reflectivity in logarithmic unit for the data assimilation. Their results showed that if the error is small, both $\mathrm{Z}_{\mathrm{H}}$ and $\mathrm{Z}_{\mathrm{DR}}$ errors can be approximated well with Gaussian distributions. For these reasons, the variational formulation can be applied for our purpose. The optimal use of radar measurements involves the minimization of the cost function $J(\mathbf{x})$, which is defined as

$$
J(\mathbf{x})=J_{b}(\mathbf{x})+J_{Z_{H}^{\prime}}(\mathbf{x})+J_{Z_{D R}^{\prime}}(\mathbf{x})+J_{K_{D P}}(\mathbf{x}),
$$

where,

$$
\begin{aligned}
& J_{b}(\mathbf{x})=\frac{1}{2}\left(\mathbf{x}-\mathbf{x}_{b}\right)^{\mathrm{T}} \mathbf{B}^{-1}\left(\mathbf{x}-\mathbf{x}_{b}\right), \\
& J_{Z_{H}^{\prime}}(\mathbf{x})=\frac{1}{2}\left[H_{Z_{H}^{\prime}}(\mathbf{x})-\mathbf{y}_{Z_{H}^{\prime}}\right]^{\mathrm{T}} \mathbf{R}_{Z_{H}^{\prime}}^{-1}\left[H_{Z_{H}^{\prime}}(\mathbf{x})-\mathbf{y}_{Z_{H}^{\prime}}\right], \\
& J_{Z_{D R}^{\prime}}(\mathbf{x})=\frac{1}{2}\left[H_{Z_{D R}^{\prime}}(\mathbf{x})-\mathbf{y}_{Z_{D R}^{\prime}}\right]^{\mathrm{T}} \mathbf{R}_{Z_{D R}^{\prime}}^{-1}\left[H_{Z_{D R}^{\prime}}(\mathbf{x})-\mathbf{y}_{Z_{D R}^{\prime}}\right], \\
& J_{K_{D P}}(\mathbf{x})=\frac{1}{2}\left[H_{K_{D P}}(\mathbf{x})-\mathbf{y}_{K_{D P}}\right]^{\mathrm{T}} \mathbf{R}_{K_{D P}}^{-1}\left[H_{K_{D P}}(\mathbf{x})-\mathbf{y}_{K_{D P}}\right],
\end{aligned}
$$

The cost function $J$ is composed of four parts as given above. $J_{b}$ represents the contribution from the background. The other three terms correspond to the observations of $Z_{\mathrm{H}}^{\prime}, Z_{\mathrm{DR}}^{\prime}$, and $K_{\mathrm{DP}}$, respectively. Superscript " $\mathrm{T}$ " denotes the matrix transpose. $\mathbf{x}$ is the state vector and $\mathbf{x}_{\mathrm{b}}$ is the background or first guess state vector. $\mathbf{y}$ indicates the radar observations. $H$ denotes the nonlinear observation operator of radar variables. $\mathbf{B}$ is the background error covariance matrix, $\mathbf{R}$ is the observational error covariance matrix. Subscripts " $Z^{\prime}{ }^{\prime}$ ", " $Z^{\prime}{ }_{\mathrm{DR}}$ " and " $K_{\mathrm{DP}}$ " are used to denote the terms for the corresponding radar observations. In the above equations, we try to follow the standard notations used in modern data assimilation literature, as defined in Ide et al. (1997).

Matrix $\mathbf{B}$ is an $m$-by- $m$ matrix where $m$ is the size of state vector $\mathbf{x}$ and is equal to the number of analysis grid points (in the 2-D region in our case) times the number of state parameters. The full matrix is usually huge. Matrix computation and storage, especially for the inversion of $\mathbf{B}$, can be a major problem during the iterative minimization of the cost function. To solve this problem, a new state variable $\mathbf{v}$ is introduced, written as,

$$
\mathbf{v}=\mathbf{D}^{-1} \delta \mathbf{x},
$$

with $\delta \mathbf{x}=\mathbf{x}-\mathbf{x}_{b}$ and $\mathbf{D D}^{\mathrm{T}}=\mathbf{B}$ (Parrish and Derber 1992). $\delta$ is the notation of the increment. $\mathbf{D}$ is the square root of matrix $\mathbf{B}$. The cost function is then rewritten as,

$$
\begin{gathered}
J(\mathbf{v})=\frac{1}{2} \mathbf{v}^{\mathrm{T}} \mathbf{v}+\frac{1}{2}\left[H_{Z_{H}^{\prime}}\left(\mathbf{x}_{b}+\mathbf{D v}\right)-\mathbf{y}_{Z_{H}^{\prime}}\right]^{\mathrm{T}} \mathbf{R}_{Z_{H}^{\prime}}^{-1}\left[H_{Z_{H}^{\prime}}\left(\mathbf{x}_{b}+\mathbf{D v}\right)-\mathbf{y}_{Z_{H}^{\prime}}\right]+ \\
\frac{1}{2}\left[H_{Z_{D R}^{\prime}}\left(\mathbf{x}_{b}+\mathbf{D v}\right)-\mathbf{y}_{Z_{D R}^{\prime}}\right]^{\mathrm{T}} \mathbf{R}_{Z_{D R}^{\prime}}^{-1}\left[H_{Z_{D R}^{\prime}}\left(\mathbf{x}_{b}+\mathbf{D v}\right)-\mathbf{y}_{Z_{D R}^{\prime}}\right]+
\end{gathered}
$$




$$
\frac{1}{2}\left[H_{K_{D P}}\left(\mathbf{x}_{b}+\mathbf{D v}\right)-\mathbf{y}_{K_{D P}}\right]^{\mathrm{T}} \mathbf{R}_{K_{D P}}^{-1}\left[H_{K_{D P}}\left(\mathbf{x}_{b}+\mathbf{D v}\right)-\mathbf{y}_{K_{D P}}\right]
$$

In this way, the inversion of $\mathrm{B}$ is avoided. The minimization of cost function $J$ is achieved by searching for the minimum of cost function $J$ making use of cost function gradient $\nabla_{\mathbf{v}} J$, which is given by,

$$
\begin{aligned}
\nabla_{\mathbf{v}} J= & \mathbf{v}+\mathbf{D}^{\mathrm{T}} \mathbf{H}_{Z_{H}^{\prime}}^{\mathrm{T}} \mathbf{R}_{Z_{H}^{\prime}}^{-1}\left(\mathbf{H}_{Z_{H}^{\prime}}^{\mathrm{T}} \mathbf{D} \mathbf{v}-\mathbf{d}_{Z_{H}^{\prime}}\right)+\mathbf{D}^{\mathrm{T}} \mathbf{H}_{Z_{D R}^{\prime}}^{\mathrm{T}} \mathbf{R}_{Z_{D R}^{\prime}}^{-1}\left(\mathbf{H}_{Z_{D R}^{\prime}}^{\mathrm{T}} \mathbf{D} \mathbf{v}-\mathbf{d}_{Z_{D R}^{\prime}}\right) \\
& +\mathbf{D}^{\mathrm{T}} \mathbf{H}_{K_{D P}}^{\mathrm{T}} \mathbf{R}_{K_{D P}}^{-1}\left(\mathbf{H}_{K_{D P}}^{\mathrm{T}} \mathbf{D} \mathbf{v}-\mathbf{d}_{K_{D P}}\right) .
\end{aligned}
$$

Here $\mathbf{H}$ represents the Jacobian operator, a matrix containing the partial derivative of observation operator $H$ with respective to each element of the state vector; it is often referred to as the linearized observation operator. $\mathbf{d}$ is the innovation vector of the observations, i.e., $\mathbf{d}=\mathbf{y}-H\left(\mathbf{x}_{\mathrm{b}}\right)$.

The spatial influence of the observation is determined by the background error covariance matrix B. Huang (2000) showed that the element $b_{\mathrm{ij}}$ of matrix B could be modeled using a Gaussian correlation model,

$$
b_{i j}=\sigma_{b}^{2} \exp \left[-\frac{1}{2}\left(\frac{r_{i j}}{r_{L}}\right)^{2}\right],
$$

where subscripts $i, j$ denote two grid points in the analysis space and $\sigma_{b}^{2}$ is the background error covariance. $r_{i j}$ is the distance between the $i^{\text {th }}$ and $j^{\text {th }}$ grid points and $r_{L}$ is the spatial decorrelation length of the background error. In this study, $r_{L}$ is assumed to be constant $(2-4 \mathrm{~km})$ in the two-dimensional analysis space, i.e., the error covariance is spatially homogeneous on the horizontal plane, as is for the isotropic covariance option in Liu and Xue (2006). The square root of $\mathbf{B}, \mathbf{D}$, can be computed by applying a recursive filter (Hayden and Purser 1995) described by Gao et al. (2004) and Liu et al. (2007). In this way, the cost of computation and storage can be reduced significantly (by a factor of B's dimension), compared to the computation of the inversion of $\mathbf{B}$.

\section{b. Forward observation operator}

In this study, the state variables to be variationally retrieved or estimated are the parameters in the assumed DSD model. The modified gamma distribution (Ulbrich, 1983)

$$
N(D)=N_{0} D^{\mu} \exp (-\Lambda D),
$$

is commonly used to model DSDs, where $N_{0}$ is the intercept parameter, $\mu$ the shape parameter and $\Lambda$ the slope parameter of the gamma DSD. In this study, we apply the gamma DSD model with a constraining relation derived by Cao et al. (2008),

$$
\mu=-0.0201 \Lambda^{2}+0.902 \Lambda-1.718 \text {. }
$$

This relation is an update of the constraint-gamma (C-G) DSD model proposed by Zhang et al. (2001) through based on 2 years' DSD data collected in central Oklahoma. The C-G DSD model, which reduces the number of free parameters from 3 to 2 , has been successfully used in direct DSD retrievals from radar measured $Z_{\mathrm{H}}$ and $Z_{\mathrm{DR}}$ (e.g., Brandes et al. 2004; Cao et al. 2008). It is used for testing our variational retrieval here. In our formulation, $N_{0}{ }^{*}=\log _{10}\left(N_{0}\right)$ and $\Lambda$ are chosen as the two state variables, thus the state vector $\mathbf{x}$ is composed of $N_{0}{ }^{*}$ and $\Lambda$ at all grid points.

Given the two DSD parameters at each grid point, the DSD can be determined. 
Subsequently, radar variables including intrinsic $Z_{H}$ and $Z_{D R}$ as well as $K_{D P}$ can be calculated. Forward operators of $Z_{H}, Z_{D R}$ and $K_{D P}$ are given by Zhang et al. (2001),

$$
\begin{aligned}
& Z_{H, V}=\frac{4 \lambda^{4}}{\pi^{4}\left|K_{w}\right|^{2}} \int_{0}^{\infty}\left|f_{H, V}(\pi)\right|^{2} N(D) d D, \quad\left(\mathrm{~mm}^{6} \mathrm{~m}^{-3}\right) \\
& Z_{D R}=\log _{10} \frac{Z_{H}}{Z_{V}}, \quad(\mathrm{~dB})
\end{aligned}
$$

and

$$
\left.K_{D P}=\frac{180 \lambda}{\pi} \int_{0}^{\infty} \operatorname{Re}\left[f_{H}(0)-f_{V}(0)\right] N(D) d D, \quad(\operatorname{deg~km})^{-1}\right)
$$

where $f_{\mathrm{H}}(\pi)$ and $f_{\mathrm{V}}(\pi)$ represent the backscattering amplitudes at horizontal and vertical polarizations, respectively. Similarly, $f_{\mathrm{H}}(0)$ and $f_{\mathrm{V}}(0)$ represent forward scattering amplitudes. $\lambda$ is the wavelength. $K_{\mathrm{w}}=\left(\varepsilon_{\mathrm{r}}-1\right) /\left(\varepsilon_{\mathrm{r}}+2\right)$ and $\varepsilon_{\mathrm{r}}$ is the complex dielectric constant of water. $\operatorname{Re}[$ ] denotes the real part of a complex value. The scattering amplitudes $f_{\mathrm{H}, \mathrm{V}}(0 / \pi)$ are calculated based on the T-matrix method. The temperature is assumed to be $10^{\circ} \mathrm{C}$ for the calculation of dielectric constant of water. The axis ratio relation of raindrop in Brandes et al. (2002) is applied. The canting angle is assumed to be zero degree for the raindrop. For computational efficiency, pre-calculated values of the scattering amplitudes are stored in a lookup table for raindrop diameters from 0.1 to 8.0 $\mathrm{mm}$ and they are used in numerical integrations in above equations.

Specific attenuations at horizontal $\left(A_{\mathrm{H}}\right)$ and vertical $\left(A_{\mathrm{V}}\right)$ polarizations can be calculated by

$$
A_{H, V}=4.343 \times 10^{3} \int_{0}^{\infty} \sigma_{e x t}^{H, V}(D) N(D) d D, \quad\left(\mathrm{~dB} \mathrm{~km}^{-1}\right)
$$

where $\sigma_{e x t}^{H, V}$ is the extinction cross section at horizontal or vertical polarizations. The specific differential attenuation $A_{\mathrm{DP}}$ is defined as,

$$
A_{D P}=A_{H}-A_{V} \quad\left(\mathrm{~dB} \mathrm{~km}^{-1}\right)
$$

If specific attenuations are known, the forward operators of $Z^{\prime}{ }_{H}$ and $Z^{\prime}{ }_{\mathrm{DR}}$ at each range gate are given by,

$$
Z_{H}^{\prime}(n)=Z_{H}(n)-2 \sum_{i=1}^{n-1} A_{H}(i) \Delta r,
$$

and, $\quad Z_{D R}^{\prime}(n)=Z_{D R}(n)-2 \sum_{i=1}^{n-1} A_{D P}(i) \Delta r$,

where numbers $i$ and $n$ denote the $i^{\text {th }}$ and $n^{\text {th }}$ range gates from the radar location, respectively. $\Delta r$ is the range resolution.

\section{c. Lookup table method}

In Eq. (5), it is expensive to directly compute the transpose of linearized operator $\mathbf{H}$, which is a matrix of partial derivatives of $H$. In general, an adjoint method is applied to compute $\mathbf{H}^{\mathrm{T}}$ efficiently without storing the full matrix. The forward operator that can be functionally represented in terms of physical parameters (e.g., Jung et al. 2008) facilitates the development of adjoint code (Errico, 1997). In this study, the calculation of radar variables [in Eqs.(8)-(10)] is based on the pre-calculated values of scattering amplitudes using the T-matrix method. This approach saves a lot of computation time but 
makes it a problem to apply an adjoint for the calculation of $\mathbf{H}^{\mathrm{T}}$. In order to solve this problem, the lookup table method is applied.

The partial derivatives of each of the polarimetric variables, i.e., $Z^{\prime}{ }_{\mathrm{H}}, Z_{\mathrm{DR}}^{\prime}$, or $K_{\mathrm{DP}}$, with respect to each of the two state variables, i.e., $\Lambda$ or $N_{0}^{*}$, are needed at each grid point. Those derivatives can be calculated using ten lookup tables of derivatives ( $\left.\frac{\partial Z_{H}}{\partial \Lambda}, \frac{\partial Z_{D R}}{\partial \Lambda}, \frac{\partial K_{D P}}{\partial \Lambda}, \frac{\partial A_{H}}{\partial \Lambda}, \frac{\partial A_{D P}}{\partial \Lambda}, \frac{\partial Z_{H}}{\partial N_{0}^{*}}, \frac{\partial Z_{D R}}{\partial N_{0}^{*}}, \frac{\partial K_{D P}}{\partial N_{0}^{*}}, \frac{\partial A_{H}}{\partial N_{0}^{*}}, \frac{\partial A_{D P}}{\partial N_{0}^{*}}\right)$. For example, from Eq. 13 the derivatives of $Z_{\mathrm{H}}^{\prime}$ (or $Z_{\mathrm{DR}}^{\prime}$ ) are calculated with the derivatives of $Z_{\mathrm{H}}$ (or $Z_{\mathrm{DR}}$ ) and $A_{\mathrm{H}}\left(\right.$ or $\left.A_{\mathrm{DP}}\right)$ by

$$
\begin{aligned}
& \frac{\partial Z_{H}^{\prime}(n)}{\partial \Lambda, o r, \partial N_{0}^{*}}=\frac{\partial Z_{H}(n)}{\partial \Lambda, o r, \partial N_{0}^{*}}-2 \sum_{i=1}^{n-1} \frac{\partial A_{H}(i)}{\partial \Lambda, o r, \partial N_{0}^{*}} \Delta r \\
& \frac{\partial Z_{D R}^{\prime}(n)}{\partial \Lambda, o r, \partial N_{0}^{*}}=\frac{\partial Z_{D R}(n)}{\partial \Lambda, o r, \partial N_{0}^{*}}-2 \sum_{i=1}^{n-1} \frac{\partial A_{D P}(i)}{\partial \Lambda, o r, \partial N_{0}^{*}} \Delta r
\end{aligned}
$$

In each lookup table, the derivative values are pre-calculated for parameter $\Lambda$ varying from 0 to 50 and parameter $N_{0}^{*}$ varying from 0 to 15 . To ensure sufficient accuracy, the range of each parameter is discretized at an interval of 0.02 . As a result, each lookup table has $2501 \times 751$ elements. In this way, the partial derivative value for operator $H$ can be found with these tables for any given values of $\Lambda$ and $N^{*}{ }_{0}$. Interpolation can be performed for values between the lookup table values of $\Lambda$ or $N^{*}{ }_{0}$ to further improve the accuracy. Generally, the estimated values in the lookup tables are sufficiently accurate for the iterative minimization of cost function because the parameter ranges are wide. For state variables out of the table range, the derivative value at the end of the range is assumed although this rarely happens in practice.

With the lookup tables, the cost for derivative calculation can be saved. Similarly, the calculations of intrinsic (i.e., non-attenuated) $Z_{\mathrm{H}}, Z_{\mathrm{DR}}, K_{\mathrm{DP}}, A_{\mathrm{H}}$, and $A_{\mathrm{DP}}$ are made efficient as well, given any two state parameters. As a result, the observational operator $H$ is computed as the combination of different values found in various lookup tables, avoiding integral operations in the forward model. Preliminary results in following sections have demonstrated that the lookup table is an efficient tool to deal with nonlinear forward models of complicated functions.

\section{d. Iteration procedure}

The iteration procedure for minimizing the cost function $J$ is shown in Fig. 1. At the beginning of the retrieval program, necessary data files such as all lookup tables, the background state parameters, and radar measured $Z^{\prime}{ }_{\mathrm{H}}, Z_{\mathrm{DR}}^{\prime}, K_{\mathrm{DP}}$, and signal-to-noise-ratio (SNR) are loaded. In the mean time, initial parameters of the variational scheme are configured. For the purpose of data quality control, only the radar measurements with $\mathrm{SNR}>1 \mathrm{~dB}$ are used in the analysis region. Additional weights that account for the data quality are added to the observation error covariance matrices (i.e., Rs). The weight is set to 1 for $\mathrm{SNR}>20 \mathrm{~dB}, 2$ for $\mathrm{SNR}>10 \mathrm{~dB}, 4$ for $\mathrm{SNR}>5 \mathrm{~dB}$ and 8 for $\mathrm{SNR}<5 \mathrm{~dB}$, respectively.

The initial state vector of variational retrieval equals to the background state vector (i.e., $\mathbf{x}=\mathbf{x}_{\mathrm{b}}$ ) and the iteration starts with $\mathbf{v}=0$. Based on the state vector, radar variables, $Z_{\mathrm{H}}, Z_{\mathrm{DR}}, K_{\mathrm{DP}}, A_{\mathrm{H}}$, and $A_{\mathrm{DP}}$, can be calculated at each grid point using the 
forward operator as well as the lookup tables of scattering amplitudes. After interpolating these radar variables from grid points to observation points, attenuated $Z_{\mathrm{H}}^{\prime}$ and $Z_{\mathrm{DR}}^{\prime}$ can then be calculated according to Eq. (13). $Z_{\mathrm{H}}^{\prime}, Z_{\mathrm{DR}}^{\prime}$ and $K_{\mathrm{DP}}$ based on state vector calculation and from real data are then used in Eq. (5) to calculate the gradient of cost function. The state vector is modified according to the innovation vector of observation and repeats the calculation of radar variables for the next iteration. The update of state vector continues during the search for minimum gradient of cost function and until the convergence of the iteration. The background state vector provides the first guess of DSD parameters for the whole variational retrieval. To avoid the information from observations to be excessively utilized, the background should not be directly derived from the observations. For simplicity, a constant state vector (i.e., uniform distribution of DSD parameters in the analysis region) is usually applied as the initial first guess. However, if the observations have data quality issue in some area where adjacent observations have little influence, more informative background will be desirable to compensate the observations in this area. After the minimization process ends in a convergence, the analysis field of DSD parameters is obtained. It is noted that the analysis result from the first convergence might not be satisfactory. To improve the retrieval, the analysis result from the first convergence is used as a new background to repeat the analysis (i.e., iteration) process. This kind of repetition, which applies the previous analysis result as the background for a new analysis, is regarded as "an outer loop" of iteration. In general, several outer loops alone would give a satisfactory analysis result, which has a relatively small cost function.

\section{Testing of algorithm using simulated data}

Tests with simulated radar data can help quantify the performance of algorithm with less uncertainty than using real observations. In this section, measurements from Xband and C-band polarimetric radars are simulated from real data of an S-band polarimetric radar, named KOUN and located at Norman, Oklahoma. Figure 2 shows an example of radar reflectivity measured by KOUN at 1948 UTC on 24 April 2011. Four "cross" marks in the figure indicate the locations of two X-band CASA IP1 radars, named KSAO and KRSP, the S-band NEXRAD KTLX radar and the C-band OU-PRIME (University of Oklahoma - Polarimetric Radar for Innovations in Meteorology and Engineering) radar. They are located at Chickasha, Rush Springs, Cleveland and Norman in Oklahoma, respectively. $Z_{\mathrm{H}}$ and $Z_{\mathrm{DR}}$ measurements of KOUN have an azimuthal resolution of one degree and a range resolution of 250 meters. They are assumed to be free of precipitation attenuation and used to simulate $\mathrm{X} / \mathrm{C}$-band radar data that contain attenuation. The azimuthal resolutions of the three $\mathrm{X} / \mathrm{C}$-band radars are assumed to be one degree and range resolutions are assumed to be 96 meters and 125 meters for CASA IP1 and OU-PRIME radars, respectively. With the simulated data, the proposed variational retrieval algorithm is evaluated

\section{a. Simulation of attenuated PRD}

Figure 3 shows the procedure of radar data simulation. S-band radar measurements are assumed to be free of attenuation and represent the intrinsic radar measurements of precipitation. S-band $Z_{\mathrm{H}}$ and $Z_{\mathrm{DR}}$ data are first interpolated to a highresolution Cartesian grid and these fields are used to generate $\mathrm{X}$-band truth and verify the 
analyses. They are also interpolated into $\mathrm{X} / \mathrm{C}$-band radar grids (at one degree azimuthal resolution and 96 or 125 meters range resolution). The interpolated $\mathrm{S}$-band $Z_{\mathrm{H}}$ and $Z_{\mathrm{DR}}$ are then used to retrieve the DSDs of rainfall, which are assumed to be measured by $\mathrm{X} / \mathrm{C}$ band radars within their observable regions. The DSD retrieval here needs to solve equation (9) using a two-parameter DSD model, e.g., exponential model or C-G model. Next, the retrieved DSD is used to calculate $\mathrm{X} / \mathrm{C}$-band radar variables using equations (9)-(12) as well as scattering amplitudes of raindrop, which have been calculated and stored in the lookup tables. Those calculated variables are assumed to be the "truth" of the $\mathrm{X} / \mathrm{C}$-band radar measurements. The attenuated $\mathrm{X} / \mathrm{C}$-band $Z_{\mathrm{H}}^{\prime}$ and $Z_{\mathrm{DR}}^{\prime}$ are then obtained from equation (13). Finally, fluctuation error and bias are added to the attenuated $Z_{\mathrm{H}}^{\prime}$ and $Z_{\mathrm{DR}}^{\prime}$ to simulate the $\mathrm{X} / \mathrm{C}$-band radar observations for the analysis of proposed retrieval algorithm. The configuration of noise and bias can vary for different experiments.

\section{b. Results of variational analysis}

Figure 4 shows one example of variational retrieval results using simulated $\mathrm{X}$ band radar observations. The simulation applies the S-band dataset shown in Fig. 2. The Gaussian errors added to the simulated X-band data $\left(Z_{\mathrm{H}}^{\prime}, Z_{\mathrm{DR}}^{\prime}, K_{\mathrm{DP}}\right)$ have standard deviations of $1 \mathrm{~dB}, 0.1 \mathrm{~dB}$, and $0.1^{\circ} / \mathrm{km}$, respectively. No biases are added to the simulated data. The initial configurations of the 2-D variational retrieval are given as follows. The analysis region is $40 \mathrm{~km}$ by $40 \mathrm{~km}$, which uses a 401 by 401 grid points in horizontal with a space of 100 meters between two adjacent grid points. The decorrelation length $r_{L}$ in Eq. (6) is set to $2 \mathrm{~km}$ in this experiment. The assumed error standard deviations for $Z^{\prime}{ }_{\mathrm{H}}, Z^{\prime}{ }_{\mathrm{DR}}$ and $K_{\mathrm{DP}}$ in the variational scheme are $1 \mathrm{~dB}, 0.1 \mathrm{~dB}$, and $0.1 \% \mathrm{~km}$, respectively, consistent with the simulated errors. A constant initial background of $\left(N_{0}{ }^{*}=3 ; \Lambda=5\right)$ is used for the variational analysis. Since the constant background does not contain much useful information, the background error is set to a large number. As shown in Fig. 4, the simulated $Z_{\mathrm{H}}^{\prime}$ and $Z_{\mathrm{DR}}^{\prime}$ of X-band KRSP radar observations have apparent attenuations, especially in the northeast region beyond $20 \mathrm{~km}$ of radar site. The simulated $Z_{\mathrm{H}}^{\prime}, Z_{\mathrm{DR}}^{\prime}$, and $K_{\mathrm{DP}}$ images appear noisy due to added errors. Because the observation operator has included the attenuation correction, the analysis results of the variational retrieval show similar features to the truth (the radar truth and simulated observations are in the radar coordinates while the analysis is on the Cartesian analysis grid). The analysis results also show smoother images than simulated observations, due to the inherent smoothing in the retrieval algorithm associated with the assumed spatial background error covariance. The smoothing helps to cancel out random errors in the observations.

Compared to the truth on analysis grids, the biases of analysis results are 0.048 $\mathrm{dB}, 0.013 \mathrm{~dB}$, and $0.007^{\circ} / \mathrm{km}$ for $Z_{\mathrm{H}}^{\prime}, Z_{\mathrm{DR}}^{\prime}$, and $K_{\mathrm{DP}}$, respectively. The root mean square errors (RMSE) are $0.837 \mathrm{~dB}, 0.109 \mathrm{~dB}$, and $0.108 \% / \mathrm{km}$ for $Z_{\mathrm{H}}^{\prime}, Z_{\mathrm{DR}}^{\prime}$, and $K_{\mathrm{DP}}$, respectively. The biases and errors of retrieval results are close to assumed biases (no bias in this example) and errors in simulated dataset. The small biases and errors also illustrate the good performance of proposed variational DSD retrieval algorithm.

Figure 5 shows another example of variational retrieval using simulated C-band radar observations. S-band dataset shown in Fig. 2 is also used for this simulation. The error standard deviations for simulated C-band data $Z_{\mathrm{H}}^{\prime}, Z_{\mathrm{DR}}^{\prime}$ and $K_{\mathrm{DP}}$ have been 
increased and are $2 \mathrm{~dB}, 0.2 \mathrm{~dB}$ and $0.2^{\circ} / \mathrm{km}$, respectively. The simulation ignores the bias as well. The analysis region is $120 \mathrm{~km}$ by $120 \mathrm{~km}$ with 601 by 601 grid points and a space of 200 meters. The decorrelation length $r_{L}$ is increased to $4 \mathrm{~km}$. The assumed errors for $Z^{\prime}{ }_{\mathrm{H}}, Z^{\prime}{ }_{\mathrm{DR}}$ and $K_{\mathrm{DP}}$ in the variational scheme are still $1 \mathrm{~dB}, 0.1 \mathrm{~dB}, 0.1^{\circ} / \mathrm{km}$, which are smaller than simulated errors. The constant background is again used for the variational analysis. Although simulated errors are higher than measurement errors assumed in the variational retrieval scheme, the algorithm still gives a good retrieval, which shows similar storm features as in the truth. The attenuation has been well corrected and the retrieved radar images have been smoothed. The biases of analysis results are $0.051 \mathrm{~dB}$, $0.071 \mathrm{~dB}$, and $-0.015^{\circ} / \mathrm{km}$, and the RMSE are $2.93 \mathrm{~dB}, 0.33 \mathrm{~dB}$, and $0.12^{\circ} / \mathrm{km}$ for $Z^{\prime}{ }_{H}$, $Z_{\mathrm{DR}}^{\prime}$, and $K_{\mathrm{DP}}$, respectively. The biases and errors of retrieval results are larger because of the increase of errors added in the simulated dataset. Although the error assumption in variational retrieval does not match the "true" error (i.e., simulated measurement error), the retrieval error is also consistent with the "true" error. The proposed retrieval algorithm still performs well for this example of C-band data.

\section{c. Sensitivity analysis}

A sensitivity analysis would help us understand the validity of the proposed variational retrieval algorithm. Several issues need to be considered for practical applications of this algorithm. Firstly, this algorithm applies a C-G DSD model and model error may exist in quantifying natural DSDs. The effect of DSD model error on the retrieval is a primary concern. Secondly, real measurement errors are always unknown and might be overestimated or underestimated in the variational scheme, as done in the example of Fig. 5. This situation should be very common in practical applications. It is therefore important to know how incorrect assumption of measurement errors would affect the retrieval. Thirdly, there might exist errors that are attributed to the forward model error. The forward model error can result in differences between radar observations and forward model outputs. Examples in Figs 4 and 5 do not include a model error although they show good performance of this algorithm for non-bias assumption. It would be helpful to know the effect of observation operator error on the retrieval.

If the data simulation assumes a different DSD from the one used in the variational analysis, the observation operator error is then introduced. For example, the 'truth' simulation may use the exponential DSD while the retrieval uses the C-G DSD model. As suggested by the study of Cao et al. (2009), however, the DSD model error does not degrade the performance of variational retrieval too much. The sensitivity tests in the rest of this section will focuses on investigating the effect of error and bias.

The sensitivity tests are based on X-band radar observations, which are simulated using the S-band KOUN radar measurements on 8 May 2007 (1230 UTC, elevation angle $0.5^{\circ}$ ) when a convective system with widespread stratiform precipitation passed through Oklahoma from west to east (Cao et al. 2009). The simulation follows the procedure described in Section 3a. In order to focus on the DSD retrieval, the analysis chooses a 20 $\mathrm{km}$ by $20 \mathrm{~km}$ region, which is mostly covered by the storm. In total 12 experiments are designed for the tests. All these tests contain DSD model error, i.e., the simulation assumes the exponential DSD while the retrieval assumes the C-G DSD. Constant background is applied in these tests. To simulate the observation, simulated radar "truth" 
is combined with different fluctuation errors and biases for 12 tests. Tests 1-4 assume no bias but different fluctuation errors for simulated observations. Tests 5-8 assume the same fluctuation errors but different biases. Tests 9-12 assume the same biases as tests 5-8 except different fluctuation errors. The detailed configurations of simulated data and error statistics of retrievals are shown in Table 1. In each cell of the table, values to the right of slash notation are simulated biases or RMSEs. Values to the left are retrieval biases or RMSEs computed against the simulated "truth". For an optimal analysis system, the observation error covariance matrix $\mathbf{R}$ should properly characterize the expected observation errors, including their magnitude and spatial correlations. The variational method based on the optimal estimation theory also assumes that all errors are unbiased (Kalnay 2002). However, the RMSEs and bias of real observations are difficult to accurately estimate. Therefore, mismatched errors are introduced in our tests to examine the sensitivity of the analysis to such error mismatches. The RMSEs of $Z_{\mathrm{H}}^{\prime}, Z_{\mathrm{DR}}^{\prime}$, and $K_{\mathrm{DP}}$ in the variational scheme are assumed to be $0.5 \mathrm{~dB}, 0.1 \mathrm{~dB}$ and $0.1 \% / \mathrm{km}$, respectively. That is to say, "measurement" errors only match the "truth" in test 1 . In other tests, "true" errors are generally larger than assumed errors in the variational scheme.

In tests 1-4, retrieval RMSE values are generally less than "true" RMSE values. This result shows that the algorithm can smooth out observation errors and result in less fluctuation error in the final analysis, consistent with the optimal estimation theory that the error of final analysis should be smaller than the error of all sources of information used (Kalnay 2002). However, the measurement error may result in retrieval bias. The bias increases with increasing the RMSE but in general the biases are very small, consistent with the fact that there is no systematic bias in the simulated measurements. Tests 5-8 have the same fluctuation errors as test 1 except they have contained different biases by adding constant values to all measurements. Compared to test 1, tests 5-8 show notable biases and RMSE values in retrieval results. Except for some values of $K_{\mathrm{DP}}$, all retrieval biases or RMSE values are larger than simulated biases or errors in tests 5-8. Test 8 shows that $1 \mathrm{~dB}$ bias in $\mathrm{Z}^{\prime}{ }_{\mathrm{H}}$ measurements leads to about $3 \mathrm{~dB}$ bias and about $3 \mathrm{~dB}$ RMSE in the $Z_{H}$ retrieval. This fact implies that the variational algorithm is more sensitive to the measurement bias than to the fluctuation error. The measurement bias not only introduces a larger bias in the retrieval but also enlarges the retrieval RMSE. Moreover, the larger the measurement bias, the larger the retrieval bias and RMSE. The larger sensitivity to measurement biases can be explained by the fact that the variational analysis assumes unbiased observations. The invalidity of this assumption can cause more problem than measurement errors themselves, and in practice every effort should be made to remove the measurement biases before the variational analysis (e.g., Harris and Kelly 2001).

Tests 9-12 have a set of measurement biases that match those of tests 5-8 but the fluctuation errors are larger. For example, the simulated data in test 12 have three times fluctuation errors as large as in test 8 . However, retrieval biases and RMSEs of test 12 are almost the same as those of test 8 . This result also demonstrates that the algorithm's sensitivity to the bias is greater than to the error. These 12 tests give us insight on the algorithm's sensitivity to the observation error and bias. In real world problems, the situation would be more complicated. For example, the expected error might not be the same for every measurement though this would not be a serious issue according to the aforementioned analysis. The serious problem might exist with systematic errors within 
different radar variables. For example, according to the radar forward model used in the retrieval algorithm, three parameters, $Z_{\mathrm{H}}, Z_{\mathrm{DR}}$, and $K_{\mathrm{DP}}$, should be intrinsically consistent. Any inconsistency is equivalent to introducing measurement biases, which might lead to large biases and RMSE values in the retrieval. Moreover, the data inconsistency might not exist everywhere equally. For example, radar measurements might not be reliable due to low SNRs in certain regions. Within the low SNR regions, measurement biases and errors might be very large while they might be small in other regions. The performance of the variational algorithm would be degraded in such a situation. With the understanding of algorithm's sensitivity, the next subsection will show some results from real radar data and discuss corresponding issues for practical implementation of the algorithm.

\section{Application to real polarimetric radar data}

The previous section tests the proposed variational algorithm using simulated data and shows promising results. The sensitivity tests also indicate that the performance of this algorithm depends on the data quality, in particular the observation bias. It is worth noting that the retrieval based on simulated data generally gives good results even though the constant background contains useless information on the measured precipitation. It makes sense because the simulated data are generally of good quality and complete data coverage in the analysis regions. In such situations, the background is not that important. However, data quality can be a major issue for real radar data. When the data quality is bad, retrievals without a good constant background are usually bad also. This section gives two cases of real data retrieval to address this issue.

The C-band OU-PRIME radar observations $\left(Z_{\mathrm{H}}^{\prime}, Z_{\mathrm{DR}}^{\prime}, K_{\mathrm{DP}}\right.$, and SNR) are shown in Fig. 6. The SNR is generally higher in the southwest region (closer to the radar) of the image while lower in other regions. The $Z^{\prime}{ }_{\mathrm{DR}}$ and $K_{\mathrm{DP}}$ are apparently noisier in the lower SNR region, where the data quality of $Z_{\mathrm{H}}^{\prime}, Z^{\prime}{ }_{\mathrm{DR}}$, and $K_{\mathrm{DP}}$ are certainly lower. For example, the southeast, northwest and west regions of $K_{\mathrm{DP}}$ image show unreasonable values, attributed to low SNRs. Using a constant background (e.g., $N_{0}{ }^{*}=3 ; \Lambda=5$ ) in the variational retrieval, the result is not satisfactory, as shown in Fig. 7 with the retrieved $Z_{\mathrm{H}}$. The retrieved $Z_{\mathrm{H}}$ only catches the major feature of the storm and the attenuation is apparently over-corrected in some parts of southwest region. This result can be explained as follows. The corrections to attenuation at different range gates of the same radar radial are interdependent. The unreliable observations in a region would affect the retrieval not only in this region but also in other regions. Additional information is therefore required to compensate the uncertainty in unreliable observations.

In the current study we take advantage of the S-band NEXRAD radar observations, which are additional observations/data sources, to assist the retrieval. Figure 8 shows the reflectivity of NEXRAD KTLX located at central Oklahoma. The speckles on this image indicate bad data there. Despite these bad data points, the integration of KTLX radar reflectivity into the retrieval scheme could improve the retrieval in Fig. 7. It is worth noting that the KTLX is the single-polarization radar. Dualpolarization information is provided by the PRD of OU-PRIME. To verify the retrieval, independent polarimetric S-band KOUN radar observations are used. The retrieval analyzes a $120 \mathrm{~km}$ by $120 \mathrm{~km}$ region (601 by 601 grids with a space of 200 meters) and assumes observation errors to be $2 \mathrm{~dB}, 0.2 \mathrm{~dB}$, and $0.2^{\circ} / \mathrm{km}$ for $Z_{\mathrm{H}}^{\prime}, Z_{\mathrm{DR}}^{\prime}$, and $K_{\mathrm{DP}}$, respectively. The decorrelation length $r_{L}$ is assumed to be $4 \mathrm{~km}$. The KTLX radar 
reflectivity is used to derive the background state vector, which assumes a MarshallPalmer DSD model. The normalized background error is assumed to be 4, i.e., 4 times as large as the observation error.

Figure 9 shows the comparison between the retrieval and KOUN observations. To make a fair comparison, KOUN observations have been converted into the C-band results using DSD retrieval described in Section 3a. As Fig. 9 shows, KOUN has a lower sensitivity than OU-PRIME and its effective precipitation region is not as large as in the retrieval results. The retrieval results generally have a good match with KOUN observations, capturing the detailed structures of precipitation, except that the retrieved $Z_{\mathrm{DR}}$ has lower values in some regions. The smoother $Z_{\mathrm{DR}}$ in the retrieval implies a balance among the factors such as data quality, error assumption, observation and background. As shown in Fig. 9, although we do not know the real error structure of data exactly, the proposed retrieval algorithm still performs well for the real data application in this example.

The next example gives an X-band real data retrieval. Similar to Figs. 6 and 9, Fig. 10 shows X-band KSAO radar observations and Fig. 11 shows the comparison of retrieval and the KOUN observations, which have been converted to X-band. The retrieval analyzes a $40 \mathrm{~km}$ by $40 \mathrm{~km}$ region (401 by 401 grids with a space of 100 meters) and the same observation errors and background error as used in the previous example. Considering the decorrelation length of $4 \mathrm{~km}$ might over-smooth the result in this case of higher data resolution, the length is set to $2 \mathrm{~km}$. As Fig. 11 shows, the X-band retrieval also has a good match with KOUN observations but while capturing more details of the storm structures in $Z_{\mathrm{H}}, Z_{\mathrm{DR}}$, and $K_{\mathrm{DP}}$. This example also illustrates the validity of the retrieval algorithm for real X-band radar data application.

\section{Summary and conclusions}

This study presents a variational approach for retrieving raindrop size distribution and associated polarimetric radar measurements using attenuated polarimetric radar data. The proposed retrieval algorithm applies a two-parameter C-G DSD model and corrects the radar attenuation simultaneously while retrieving the DSD optimally (based on optimal estimation theory). It primarily uses $Z_{\mathrm{H}}^{\prime}, Z_{\mathrm{DR}}^{\prime}$, and $K_{\mathrm{DP}}$ data of a single radar but can be easily extended to include observations from multiple radars to improve the retrieval. The verification of the retrieval focuses on the accuracy of polarimetric variables calculated from the DSD. Analyses based on simulated data show that the proposed algorithm has a great potential for radar DSD retrieval. Sensitivity experiments show that the more the error and/or bias in the observations, the higher the RMSE and bias in the retrieval. Moreover, the algorithm is more sensitive to the bias than the fluctuation error in the observation. The sensitivity analysis also indicates that radar data quality, especially with systematic biases, can be a serious issue for the real data applications. The uncertainty of retrieval in the region of low data quality can be mitigated by using additional data from other radars that provide useful precipitation information in the same region. This is also the advantage of the variational estimation framework over deterministic methods that the problem can be over-determined and the final analysis is an optimal combination of all sources of information. This study suggests the usage of the single-polarization NEXRAD radar to provide informative background in

the analysis. The real data application using X-band KSAO and C-band OU-PRIME radar 
data demonstrates the validity of the proposed algorithm with the use of KTLX radar background. It also implies the capability of the proposed algorithm applied in other radar platforms and in other regions of the United States.

The proposed variational scheme can accommodate a 3-parameter DSD model and/or more species of hydrometeors. We have considered using more complicated DSD model (3-moment DSD or PSD for different phases) through more observations from multiple radars (and/or multiple-frequency radars). This study has focused on the rainfall and used a 2-parameter DSD model because we wanted to use redundant information for the retrieval to reduce the uncertainty associated with different observation errors from radar data. To extend the capability of proposed algorithm in different atmospheric conditions, multiple hydrometeor species, such as rain, snow, hail, or melting phase, should be considered in the forward observation operator. The consideration of multiple species, however, requires more observations to resolve their microphysical structures. For example, if three species (rain, snow, melting particles) exist in the storm and a twoparameter microphysical model characterizes each species, at least six independent observations would be required for the retrieval. If there are not enough observations, the problem can be under-constrained by the observations, and the end results may depend too much on the background. There can therefore be a trade-off between the number of species to be considered and the number of model parameters to be estimated. With the success of retrieving liquid rain in this study, future work can seek to extend the variational retrieval algorithm to situations involving multiple hydrometeor species. An alternative approach is to include an atmospheric prediction model and sophisticated microphysics parameterization in the estimation system, as is done in Xue et al. (2009); the effectiveness of that approach remains to be tested with real data case, however, and does represent another line of research.

Acknowledgements This work was primarily supported by NSF grants AGS-0608168 and AGS-1046171. The third author was also supported by NSF grants OCI-0905040, AGS-0802888, AGS-0941491, and AGS-1046081. We are thankful for Dr. Terry Schuur from NOAA National Severe Storms Laboratory (NSSL) for providing KOUN data, and Dr. Jerry Brotzge from CAPS who provided CASA IP1 data. CASA is supported by NSF EEC-0313747. We also appreciate the engineers of the Atmospheric Radar Research Center (ARRC) for maintaining the OU-PRIME and collecting radar data. 


\section{Reference:}

Berne, A., and R. Uijlenhoet, 2006: Quantitative analysis of X-band weather radar attenuation correction accuracy. Nat. Hazards Earth Syst. Sci., 6, 419-425

Brandes, E. A., G. Zhang, and J. Vivekanandan, 2004: Drop size distribution retrieval with polarimetric radar: model and application. J. Appl. Meteor., 43, 461-475

Brandes, E., G. Zhang, and J. Vivekanandan, 2002: Experiments in rainfall estimation with a polarimetric radar in a subtropical environment. J. Appl. Meteor., 41, 674-685.

Bringi, V.N., and V. Chandrasekar, 2001: Polarimetric Doppler Weather Radar: Principles and Applications. Cambridge University Press.

Bringi, V.N., T.D. Keenan, and V. Chandrasekar, 2001: Correcting C-band radar reflectivity and differential reflectivity data for rain attenuation: A self-consistent method with constraints. IEEE Trans. Geosci. Remote Sens., 39, 1906-1915.

Bringi, V.N., V. Chandrasekar, N. Balakrishnan, and D. Zrnić, 1990: An Examination of Propagation Effects in Rainfall on Radar Measurements at Microwave Frequencies. $J$. Atmos. Oceanic Technol., 7, 829-840.

Cao, Q., G. Zhang, E. Brandes, T. Schuur, A. Ryzhkov, and K. Ikeda, 2008: Analysis of Video Disdrometer and Polarimetric Radar Data to Characterize Rain Microphysics in Oklahoma. J. Appl. Meteor. Climatol., 47, 2238-2255

Cao, Q., G. Zhang, and M. Xue, 2009: Variational retrieval of raindrop size distribution from polarimetric radar data in presence of attenuation. Preprint, $25^{\text {th }}$ IIPS, AMS Annual Meeting, Phoenix, AZ, 11-15 January 2009 (Available online: https://ams.confex.com/ams/pdfpapers/144407.pdf)

Cao, Q., G. Zhang, E. Brandes, and T. Schuur, 2010: Polarimetric Radar Rain Estimation through Retrieval of Drop Size Distribution Using a Bayesian Approach. J. Appl. Meteor. Climatol., 49, 973-990.

Delrieu, G., H. Andrieu, and J.D. Creutin, 2000: Quantification of Path-Integrated Attenuation for X- and C-Band Weather Radar Systems Operating in Mediterranean Heavy Rainfall. J. Appl. Meteor., 39, 840-850.

Doviak, R.J., and Zrnić, 1993: Doppler radar and Weather Observations. Academic press, 562 pages.

Errico, R.M., 1997: What Is an Adjoint Model?. Bull. Amer. Meteor. Soc., 78, 25772591.

Gao, J., M. Xue, K. Brewster, and K.K. Droegemeier, 2004: A Three-Dimensional Variational Data Analysis Method with Recursive Filter for Doppler Radars. J. Atmos. Oceanic Technol., 21, 457-469.

Gorgucci, E., and L. Baldini, 2007: Attenuation and Differential Attenuation Correction of C-Band Radar Observations Using a Fully Self-Consistent Methodology. Geoscience and Remote Sensing Letters, IEEE, 2, 326-330

Hayden C.M. and R.J. Purser, 1995: Recursive filter objective analysis of meteorological fields: Applications to NESDIS operational processing. J. Appl. Meteor., 34, 3-15.

Harris, B. A. and G. Kelly, 2001: A satellite radiance-bias correction scheme for data assimilation. Quart. J. Roy. Meteor. Soc., 127, 1453-1468.

Hogan, R.J., 2007: A Variational Scheme for Retrieving Rainfall Rate and Hail Reflectivity Fraction from Polarization Radar. J. Appl. Meteor. Climatol., 46, 15441564. 
Huang, X.Y., 2000: Variational Analysis Using Spatial Filters. Mon. Wea. Rev., 128, 2588-2600.

Ide, K., P. Courtier, M. Ghil, and A. Lorenc, 1997: Unified notation for data assimilation: Operational, sequential and variational. J. Meteor. Soc. Japan, 75, 181-189.

Jung, Y., G. Zhang, and M. Xue, 2008: Assimilation of Simulated Polarimetric Radar Data for a Convective Storm Using the Ensemble Kalman Filter. Part I: Observation Operators for Reflectivity and Polarimetric Variables. Mon. Wea. Rev., 136, 22282245.

Kalnay, E., 2002: Atmospheric Modeling, Data Assimilation, and Predictability. Cambridge University Press, 341 pp.

Koizumi, K., Y. Ishikawa, and T. Tsuyuki, 2005: Assimilation of precipitation data to the JMA mesoscale model with a four-dimensional variational method and its impact on precipitation forecasts. SOLA, 1, 45-48, 2005.

Liu, H. and M. Xue, 2006: Retrieval of moisture from slant-path water vapor observations of a hypothetical GPS network using a three-dimensional variational scheme with anisotropic background error. Mon. Wea. Rev., 134, 933-949.

Liu, H., M. Xue, R. J. Purser, and D. F. Parrish, 2007: Retrieval of moisture from simulated GPS slant-path water vapor observations using 3DVAR with anisotropic recursive filters. Mon. Wea. Rev., 135, 1506-1521.

Liu, Y., V. Bringi, and M. Maki, 2006: Improved rain attenuation correction algorithms for radar reflectivity and differential reflectivity with adaptation to drop shape model variation. Proceedings. IGARSS 2006, Denver, Colorado

Matrosov, S.Y., K.A. Clark, B.E. Martner, and A. Tokay, 2002: X-Band Polarimetric Radar Measurements of Rainfall. J. Appl. Meteor., 41, 941-952.

McLaughlin, D.J., 2010: Gap free CONUS surveillance using dense networks of short range radars. Phased Array Systems and Technology (ARRAY), 2010 IEEE International Symposium on , vol., no., pp.37-43, 12-15 Oct. 2010, doi: 10.1109/ARRAY.2010.5613393

Meneghini, R., and L. Liao, 2007: On the Equivalence of Dual-Wavelength and DualPolarization Equations for Estimation of the Raindrop Size Distribution. J. Atmos. Oceanic Technol., 24, 806-820.

Park, S.G., V.N. Bringi, V. Chandrasekar, M. Maki, and K. Iwanami, 2005: Correction of Radar Reflectivity and Differential Reflectivity for Rain Attenuation at X Band. Part I: Theoretical and Empirical Basis. J. Atmos. Oceanic Technol., 22, 1621-1632.

Parrish, D. F. and J. C. Derber, 1992: The National Meteorological Center's spectral statistical-interpolation analysis system. Mon. Wea. Rev., 120, 1747-1763. Rosenfeld, D., and C.W. Ulbrich, 2003: Cloud Microphysical Properties, Processes, and Rainfall Estimation Opportunities. Meteor. Monogr., 30, 237.

Ryzhkov, A., P. Zhang, D. Hudak, J. L. Alford, M. Knight and J.W. Conway, 2007: Validation of polarimetric methods for attenuation correction at $\mathrm{C}$ band. AMS, 33rd Conference on Radar Meteorology, Cairns, Queensland, Australia, August 2007

Ryzhkov, A., S. Giangrande, and T. Schuur, 2005a: Rainfall estimation with a polarimetric prototype of WSR-88D. J. Appl. Meteor., 44, 502-515.

Ryzhkov, A., and D. Zrnic, 1995: Precipitation and Attenuation Measurements at a 10-cm Wavelength. J. Appl. Meteor., 34, 2121-2134. 
Testud, J., E. Le Bouar, E. Obligis, and M. Ali-Mehenni, 2000: The Rain Profiling Algorithm Applied to Polarimetric Weather Radar. J. Atmos. Oceanic Technol., 17, 332-356.

Ulbrich, C.W., 1983: Natural Variations in the Analytical Form of the Raindrop Size Distribution. J. Climate Appl. Meteor., 22, 1764-1775.

Vulpiani, G., F.S. Marzano, V. Chandrasekar, and S. Lim, 2005: Constrained Iterative Technique With Embedded Neural Network for Dual-Polarization Radar Correction of Rain Path Attenuation. IEEE Trans. Geosci. Remote Sensing, 43, 2305-2314

Xue, M., M. Tong, and K. K. Droegemeier, 2006: An OSSE framework based on the ensemble square-root Kalman filter for evaluating impact of data from radar networks on thunderstorm analysis and forecast. J. Atmos. Ocean Tech., 23, 46-66.

Xue, M., M. Tong, and G. Zhang, 2009: Simultaneous state estimation and attenuation correction for thunderstorms with radar data using an ensemble Kalman filter: Tests with simulated data. Q. J. Roy. Meteor. Soc., 135, 1409-1423.

Xue, M., Y. Jung, and G. Zhang, 2007: Error modeling of simulated reflectivity observations for ensemble Kalman filter assimilation of convective storms, Geophys. Res. Lett., 34, L10802, doi:10.1029/2007GL029945.

Zhang, G., J. Vivekanandan, and M.K. Politovich, 2004: Radar/radiometer combination to retrieve cloud characteristics for icing detection. AMS, 11th Conference on Aviation, Range, and Aerospace., Hyannis, MA

Zhang, G., J. Vivekanandan, and E. Brandes, 2001: A method for estimating rain rate and drop size distribution from polarimetric radar. IEEE Trans. Geosci. Remote Sensing, 39, 830-840.

Figure Captions:

Fig. 1 Flowchart of the variational DSD retrieval scheme. A detailed description is given in section 2a.

Fig. 2. (a) Radar reflectivity ( 0.5 degree elevation angle) and (b) Differential reflectivity measured by KOUN radar at 1948 UTC on 24 April 2011. The "cross" marks indicate the locations of X-band KSAO and KRSP radars, C-band OU-PRIME radar and S-band KTLX radar. $40 \mathrm{~km}$ range rings are for KSAO and KRSP radar. $120 \mathrm{~km}$ range ring is for OU-PRIME radar.

Fig. 3. The procedure of C-/X-band radar data simulation based on S-band radar data.

Fig. 4. Retrieval validation using X-band KRSP radar data simulated from S-band KOUN data measured at 1948 UTC on 24 April 2011. Three rows show analysis results, simulated X-band radar observations, and simulation truth, respectively. Three columns from left to right show radar reflectivity, differential reflectivity, and specific differential phase, respectively.

Fig. 5. Retrieval validation using C-band OU-PRIME radar data simulated from S-band KOUN data measured at 1948 UTC on 24 April 2011. Three rows show analysis results, simulated C-band radar observations, and simulation truth, respectively. Three columns from left to right show radar reflectivity, differential reflectivity, and specific differential phase, respectively.

Fig. 6. C-band OU-PRIME radar observations: a) radar reflectivity; b) differential reflectivity; c) specific differential phase; and d) SNR ( 0.5 degree elevation angle, 1950 UTC on 24 April 2011). 
Fig. 7. Reflectivity calculated from DSDs retrieved from PRD shown in Fig. 6. The retrieval applies a constant background, which results in a bad retrieval.

Fig. 8. S-band NEXRAD radar reflectivity (KTLX radar, single-polarization, 0.5 degree elevation angle, 1948 UTC, 24 April 2011).

Fig. 9. Comparison of retrieval results (left column) and simulation results from S-band KOUN data (right column). The retrieval is based on C-band OU-PRIME data shown in Fig. 6. Three rows from top to bottom show radar reflectivity, differential reflectivity, and specific differential phase, respectively.

Fig. 10. X-band CASA IP1 KSAO radar observations: a) radar reflectivity; b) differential reflectivity; c) specific differential phase; and d) SNR (2 degree elevation angle, 1950 UTC on 24 April 2011).

Fig. 11. Comparison of retrieval results (left column) and simulation results from S-band KOUN data (right column). The retrieval is based on X-band KSAO data shown in Fig. 10. Three rows from top to bottom show radar reflectivity, differential reflectivity, and specific differential phase, respectively. 
Table 1: Bias and RMSE of variational retrieval for different experiments

\begin{tabular}{|c|c|c|c|c|c|c|}
\hline & \multicolumn{3}{|c|}{ Retrieval bias / Simulated bias } & \multicolumn{3}{c|}{ Retrieval RMSE / Simulated error } \\
\hline TEST & $Z_{\mathrm{H}}(\mathrm{dB})$ & $\mathrm{Z}_{\mathrm{DR}}(\mathrm{dB})$ & $\mathrm{K}_{\mathrm{DP}}(\mathrm{deg} / \mathrm{km})$ & $\mathrm{Z}_{\mathrm{H}}(\mathrm{dB})$ & $\mathrm{Z}_{\mathrm{DR}}(\mathrm{dB})$ & $\mathrm{K}_{\mathrm{DP}}(\mathrm{deg} / \mathrm{km})$ \\
\hline 1 & $0.091 / 0$ & $0.027 / 0$ & $0.004 / 0$ & $0.393 / 0.5$ & $0.107 / 0.1$ & $0.084 / 0.1$ \\
\hline 2 & $0.083 / 0$ & $0.009 / 0$ & $0.006 / 0$ & $0.409 / 1.0$ & $0.108 / 0.2$ & $0.083 / 0.2$ \\
\hline 3 & $0.178 / 0$ & $0.023 / 0$ & $0.012 / 0$ & $0.476 / 1.5$ & $0.110 / 0.3$ & $0.088 / 0.3$ \\
\hline 4 & $0.267 / 0$ & $0.036 / 0$ & $0.019 / 0$ & $0.537 / 2.0$ & $0.120 / 0.4$ & $0.093 / 0.4$ \\
\hline 5 & $0.440 / 0.125$ & $0.115 / 0.025$ & $0.020 / 0.025$ & $0.597 / 0.5$ & $0.159 / 0.1$ & $0.084 / 0.1$ \\
\hline 6 & $0.841 / 0.25$ & $0.219 / 0.05$ & $0.037 / 0.05$ & $0.952 / 0.5$ & $0.253 / 0.1$ & $0.092 / 0.1$ \\
\hline 7 & $1.575 / 0.5$ & $0.411 / 0.1$ & $0.067 / 0.1$ & $1.687 / 0.5$ & $0.445 / 0.1$ & $0.114 / 0.1$ \\
\hline 8 & $2.879 / 1.0$ & $0.755 / 0.2$ & $0.118 / 0.2$ & $3.037 / 0.5$ & $0.807 / 0.1$ & $0.160 / 0.1$ \\
\hline 9 & $0.448 / 0.125$ & $0.113 / 0.025$ & $0.022 / 0.025$ & $0.606 / 0.75$ & $0.157 / 0.15$ & $0.085 / 0.15$ \\
\hline 10 & $0.862 / 0.25$ & $0.216 / 0.05$ & $0.040 / 00.05$ & $0.979 / 1.0$ & $0.250 / 0.2$ & $0.095 / 0.2$ \\
\hline 11 & $1.604 / 0.5$ & $0.408 / 0.1$ & $0.071 / 0.1$ & $1.724 / 1.25$ & $0.443 / 0.25$ & $0.117 / 0.25$ \\
\hline 12 & $2.940 / 1.0$ & $0.747 / 0.2$ & $0.122 / 0.2$ & $3.117 / 1.5$ & $0.801 / 0.3$ & $0.165 / 0.3$ \\
\hline
\end{tabular}




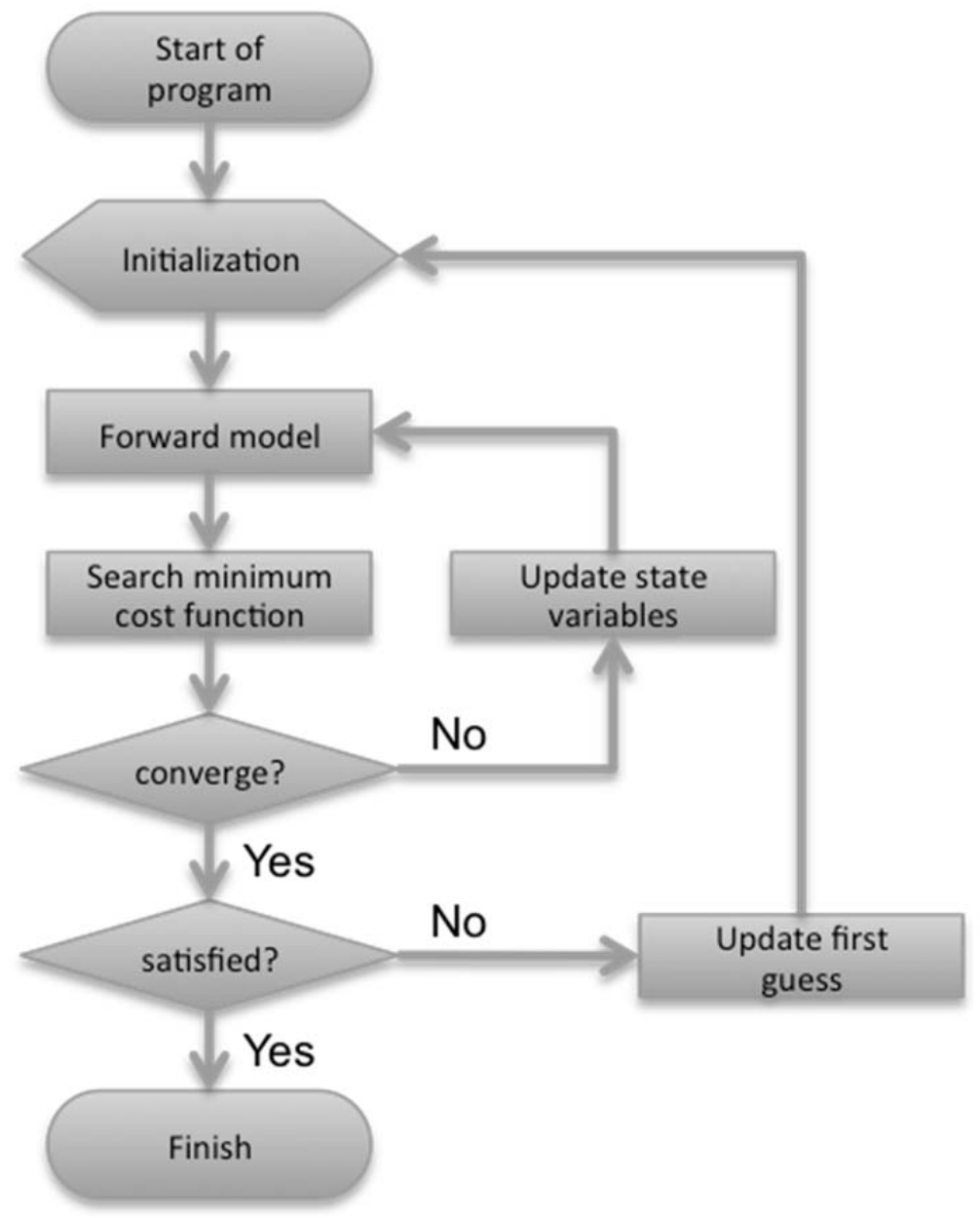

Fig. 1 Flowchart of the variational DSD retrieval scheme. A detailed description is given in section 2a. 
(a)

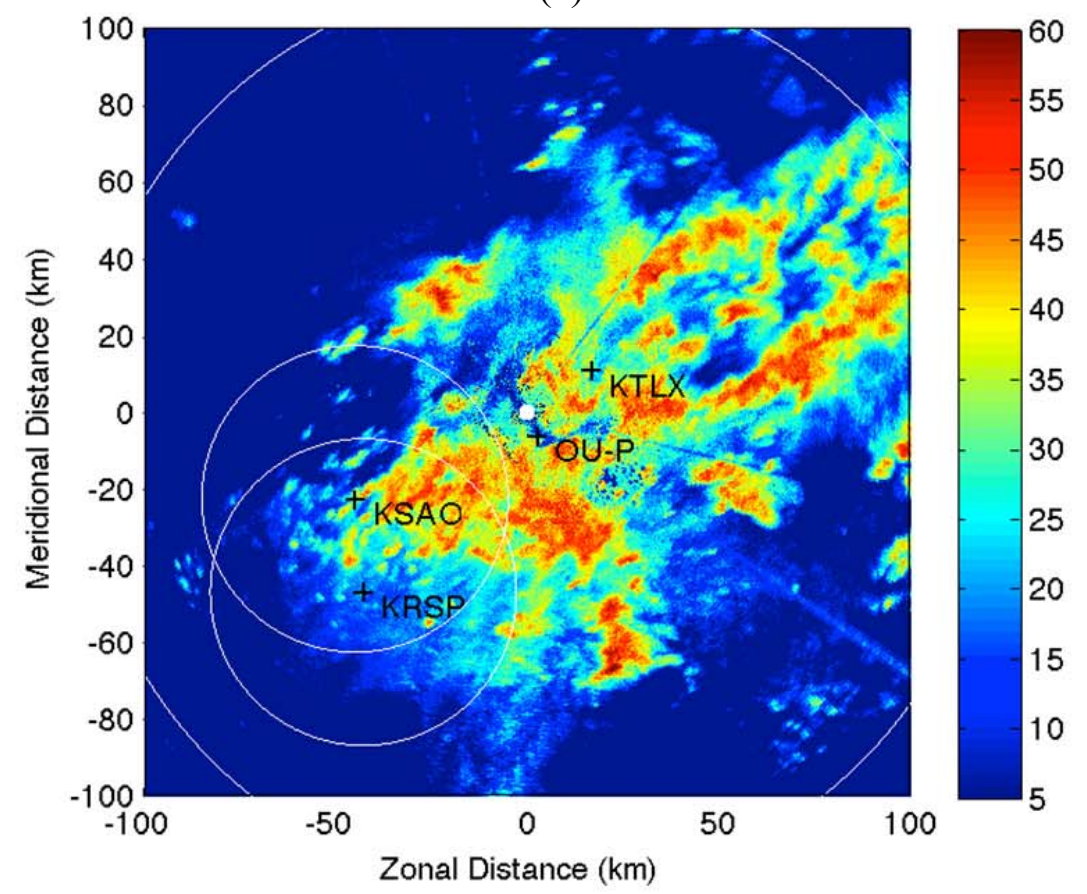

(b)

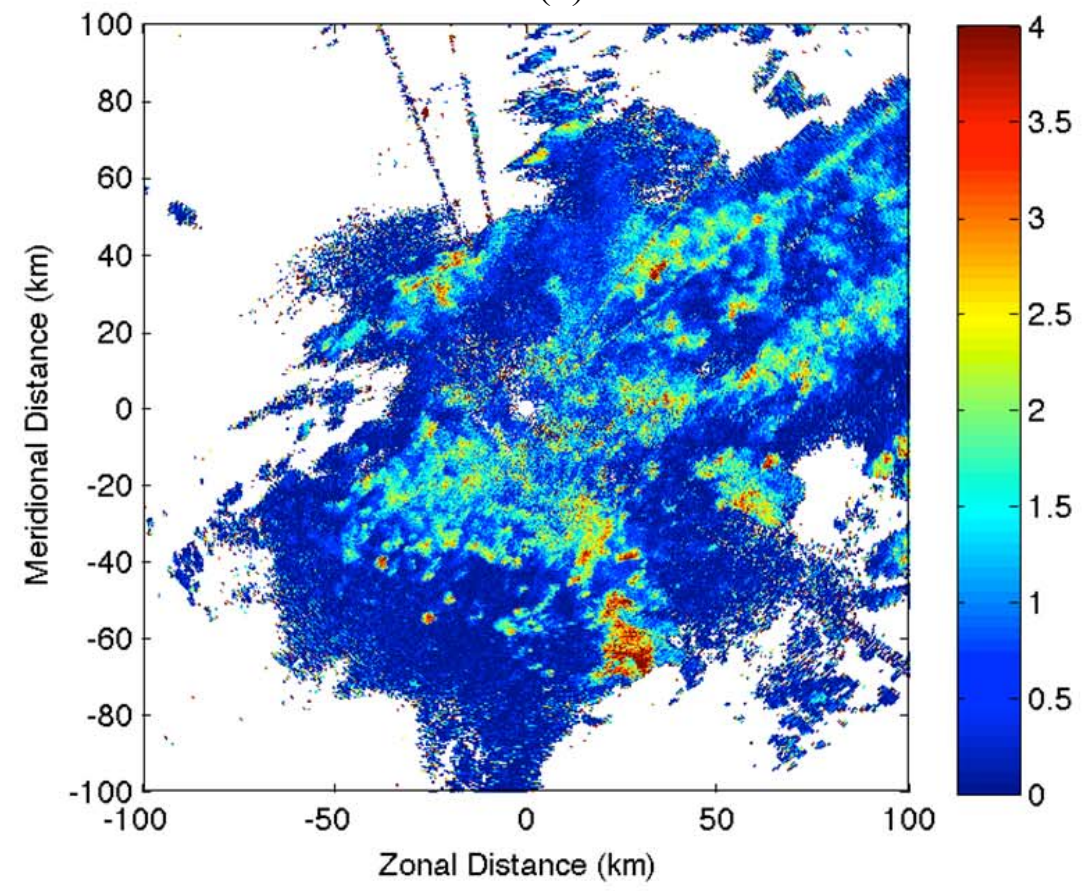

Fig. 2. (a) Radar reflectivity ( 0.5 degree elevation angle) and (b) Differential reflectivity measured by KOUN radar at 1948 UTC on 24 April 2011. The "cross" marks indicate the locations of X-band KSAO and KRSP radars, C-band OU-PRIME radar and S-band KTLX radar. $40 \mathrm{~km}$ range rings are for KSAO and KRSP radar. $120 \mathrm{~km}$ range ring is for OU-PRIME radar. 


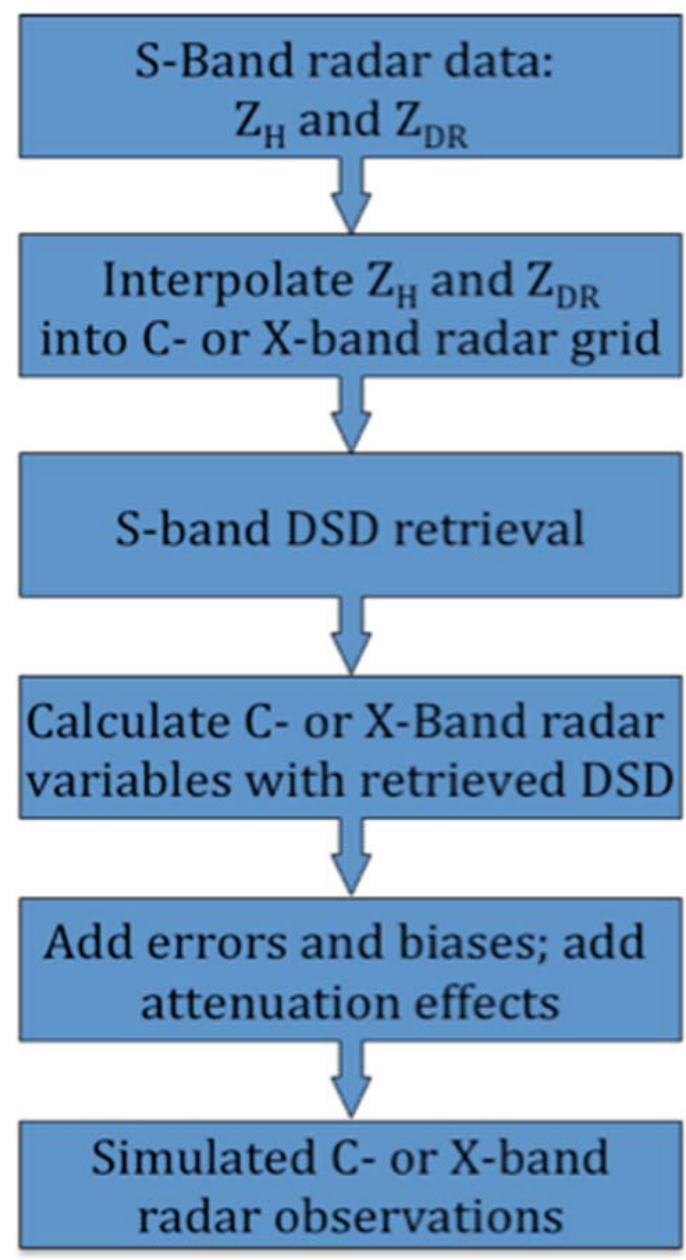

Fig. 3. The procedure of C-/X-band radar data simulation based on S-band radar data. 

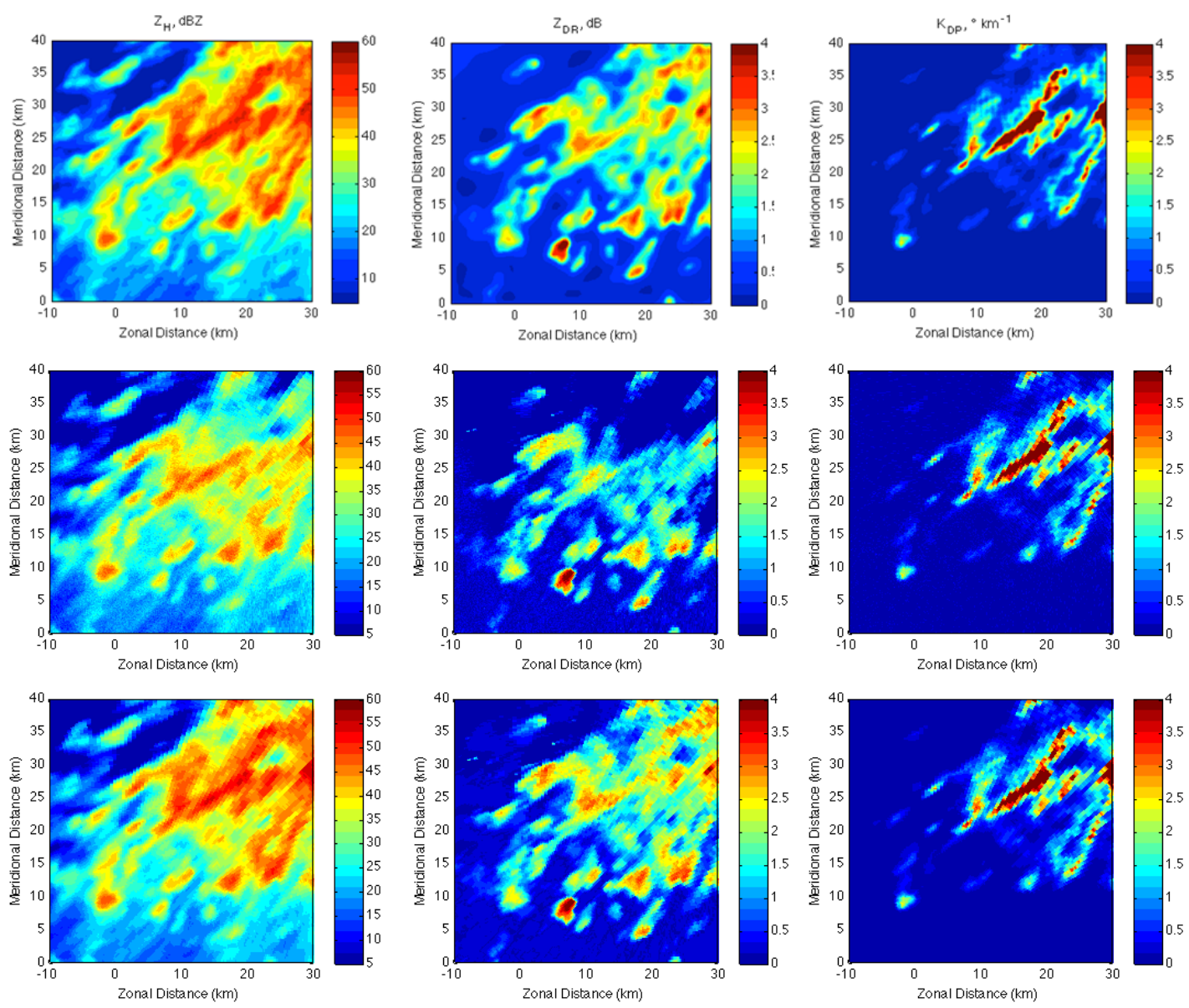

Fig. 4. Retrieval validation using X-band KRSP radar data simulated from S-band KOUN data measured at 1948 UTC on 24 April 2011. Three rows show analysis results, simulated X-band radar observations, and simulation truth, respectively. Three columns from left to right show radar reflectivity, differential reflectivity, and specific differential phase, respectively. 

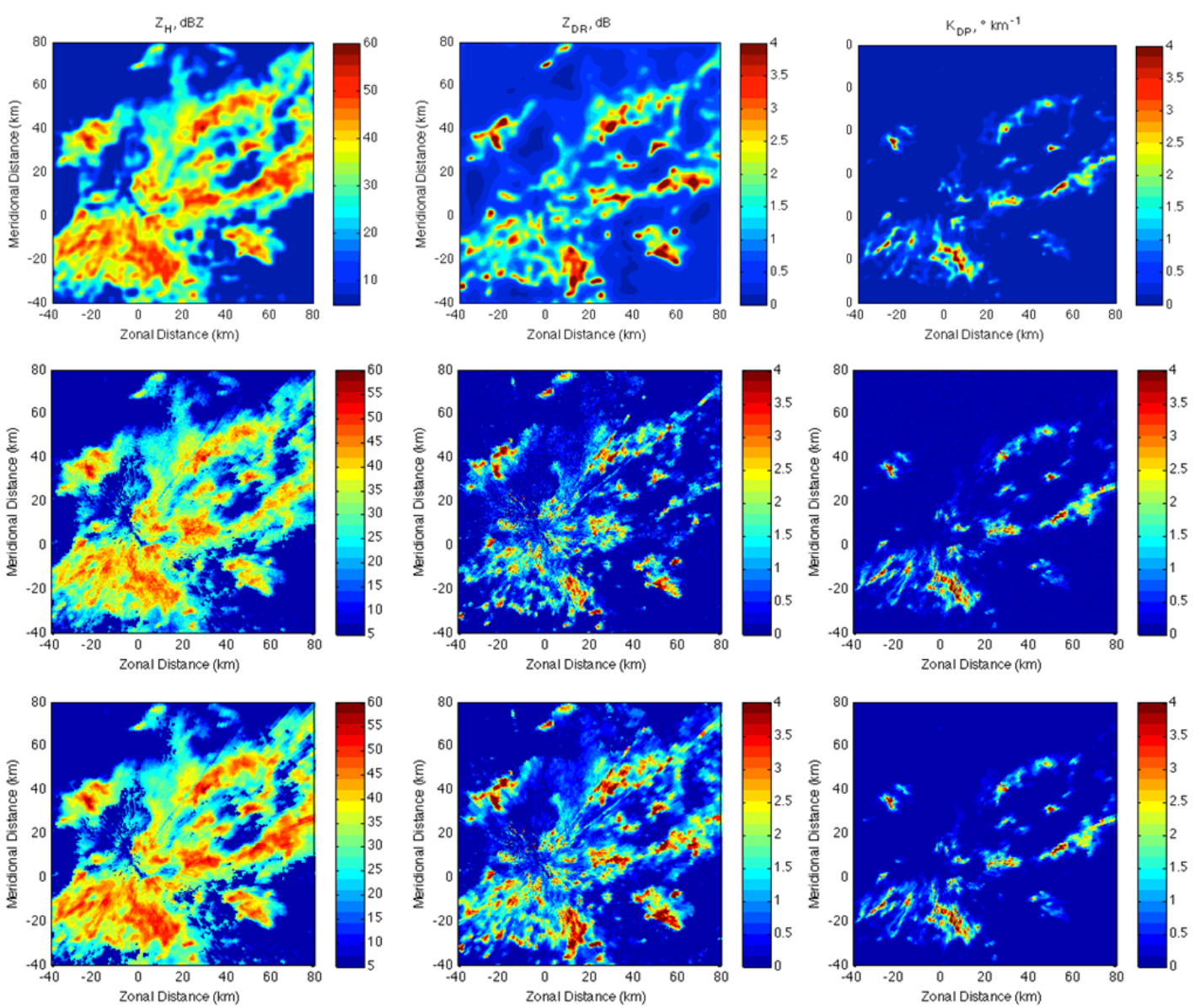

Fig. 5. Retrieval validation using C-band OU-PRIME radar data simulated from S-band KOUN data measured at 1948 UTC on 24 April 2011. Three rows show analysis results, simulated C-band radar observations, and simulation truth, respectively. Three columns from left to right show radar reflectivity, differential reflectivity, and specific differential phase, respectively. 
(a) $z_{H}, d B Z$

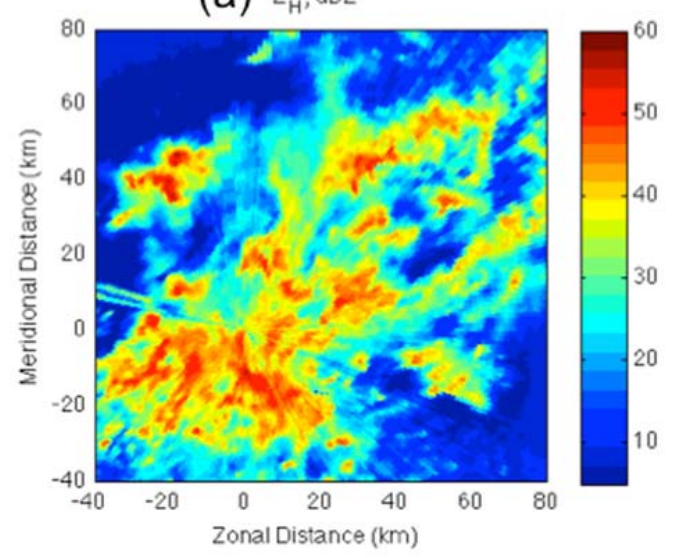

(c) $\mathrm{K}_{\mathrm{Dp},} \cdot \mathrm{km}^{-1}$

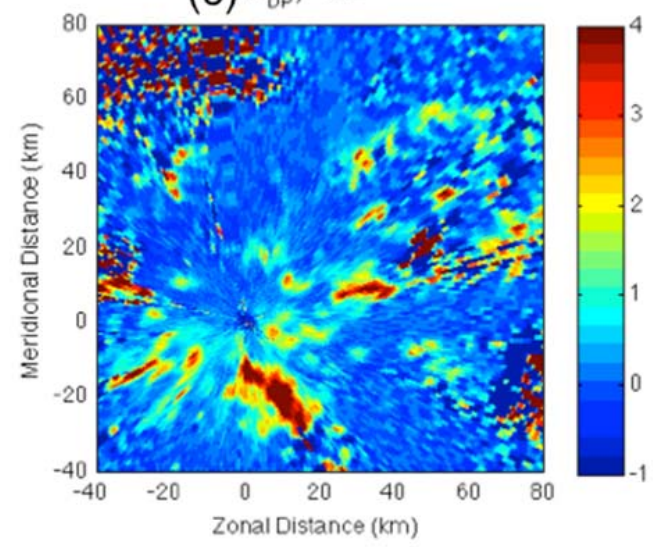

(b) $z_{D R}, d B$

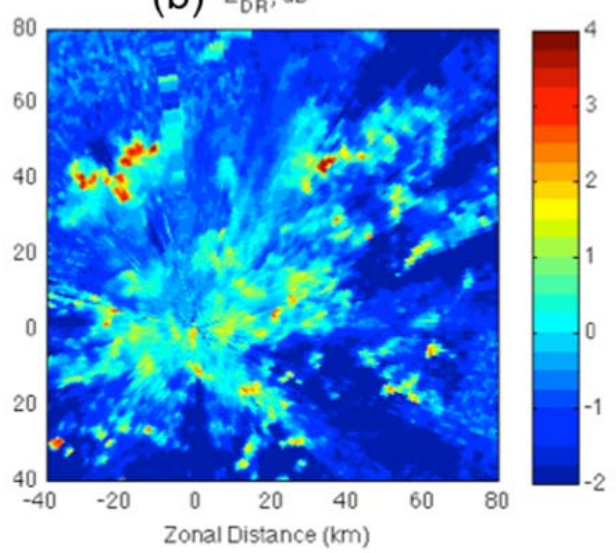

(d) $S N R, d B$

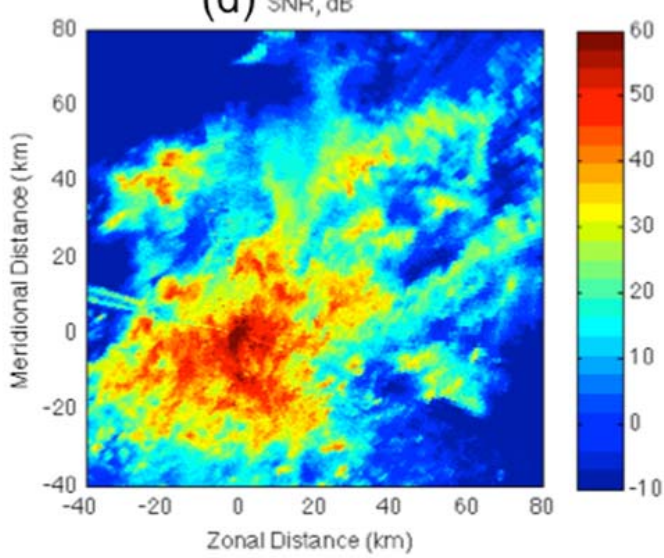

Fig. 6. C-band OU-PRIME radar observations: a) radar reflectivity; b) differential reflectivity; c) specific differential phase; and d) SNR (0.5 degree elevation angle, 1950 UTC on 24 April 2011). 


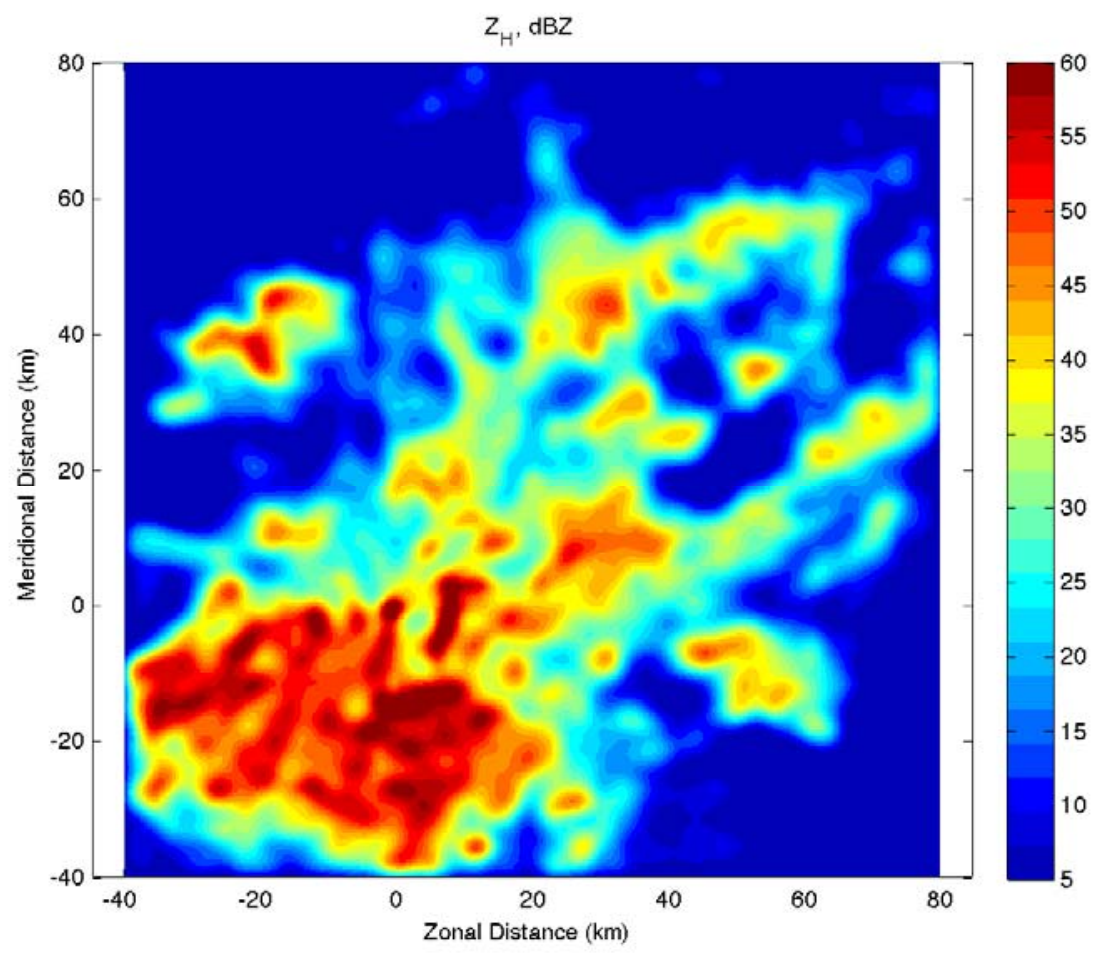

Fig. 7. Reflectivity calculated from DSDs retrieved from PRD shown in Fig. 6. The retrieval applies a constant background, which results in a bad retrieval. 


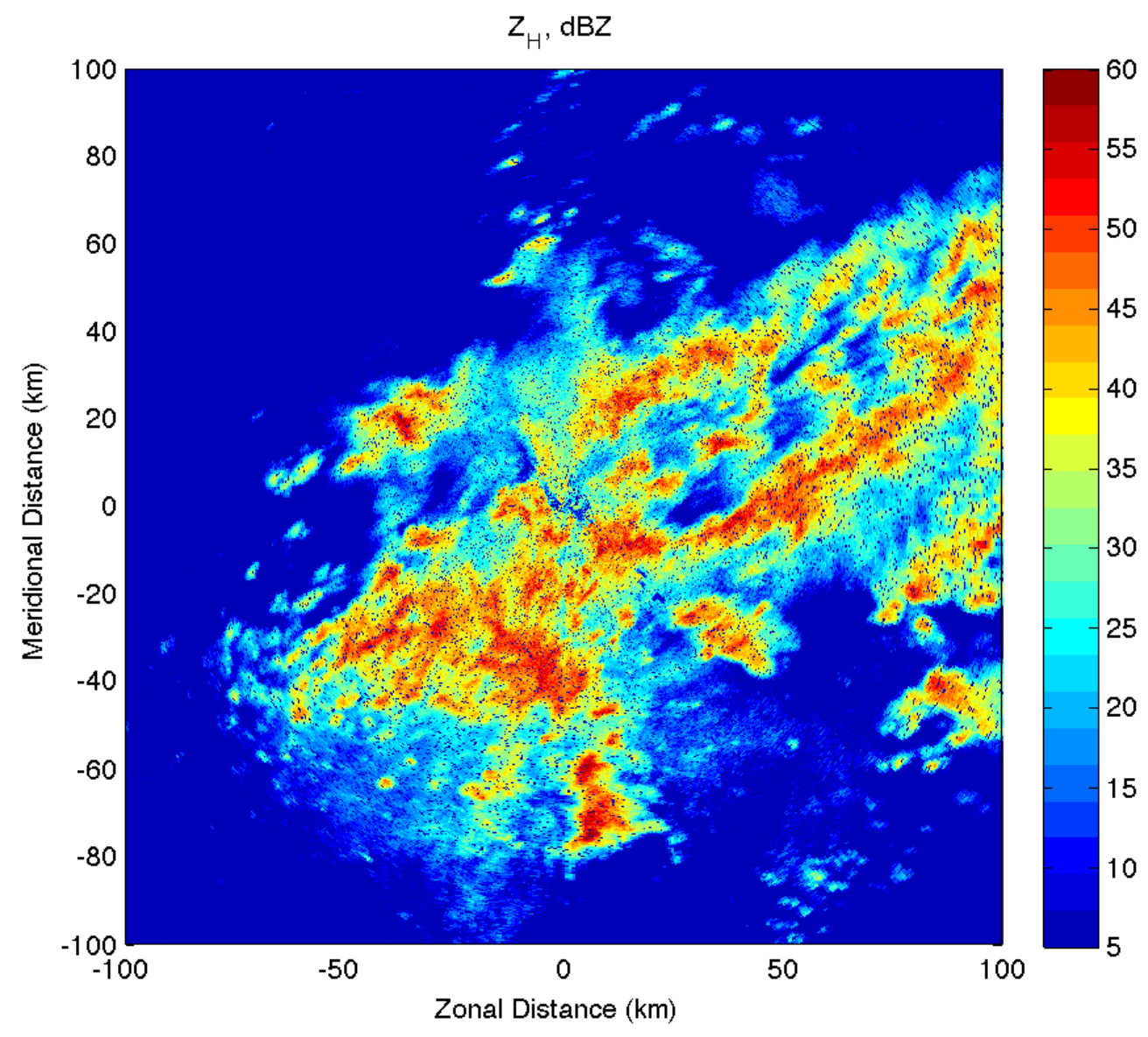

Fig. 8. S-band NEXRAD radar reflectivity (KTLX radar, single-polarization, 0.5 degree elevation angle, 1948 UTC, 24 April 2011). 

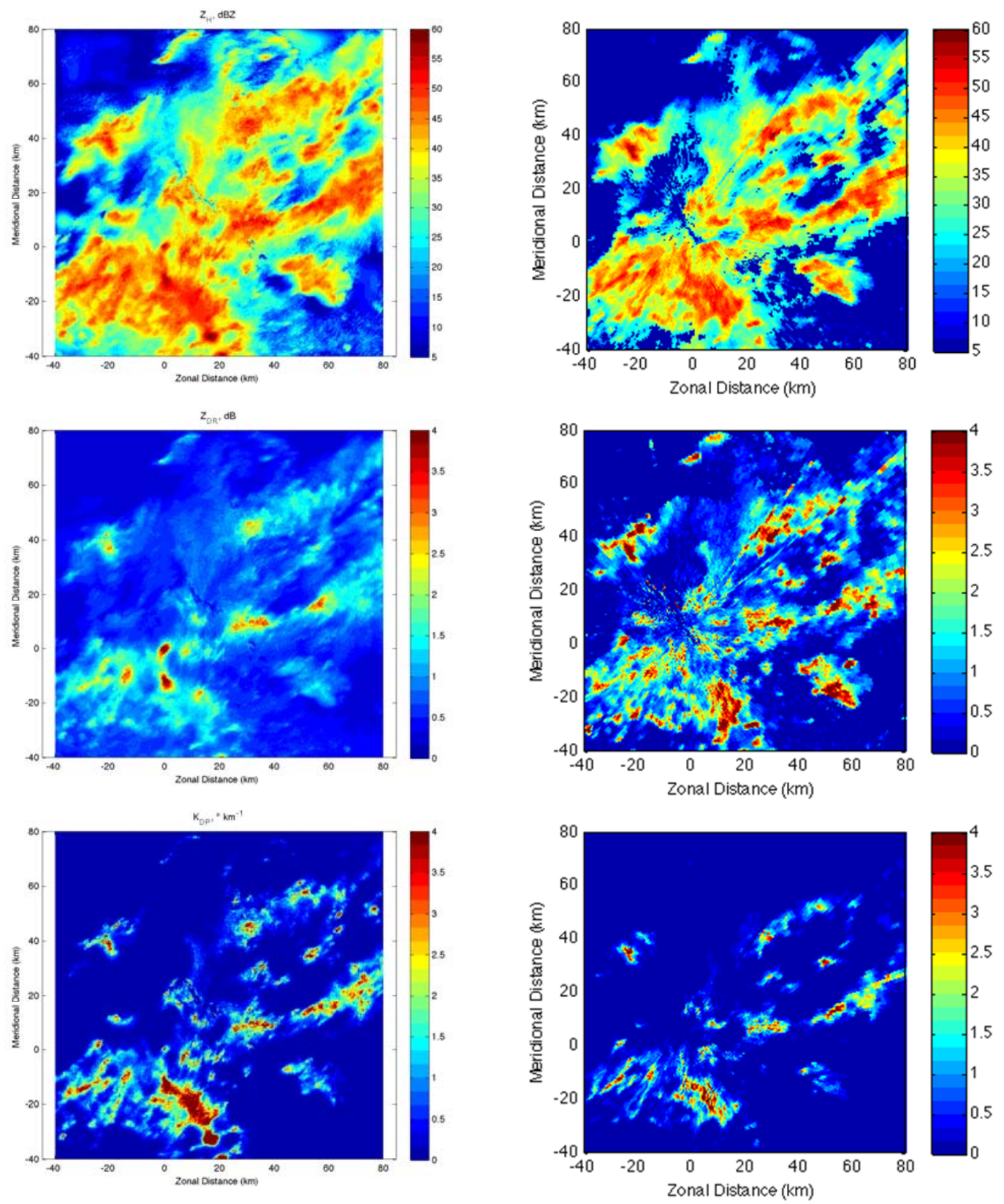

Fig. 9. Comparison of retrieval results (left column) and simulation results from S-band KOUN data (right column). The retrieval is based on C-band OU-PRIME data shown in Fig. 6. Three rows from top to bottom show radar reflectivity, differential reflectivity, and specific differential phase, respectively.

(a) 


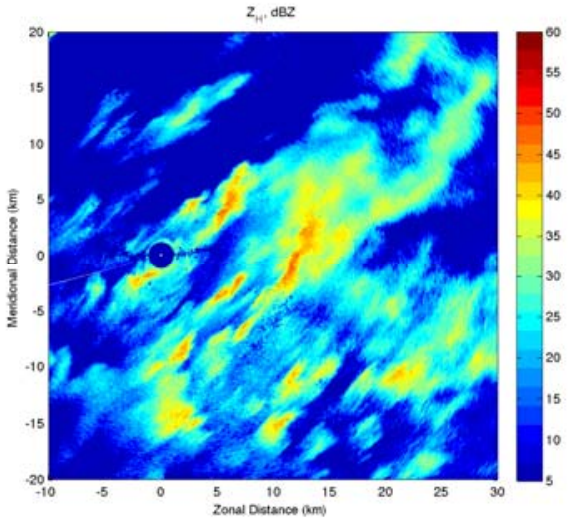

(c)

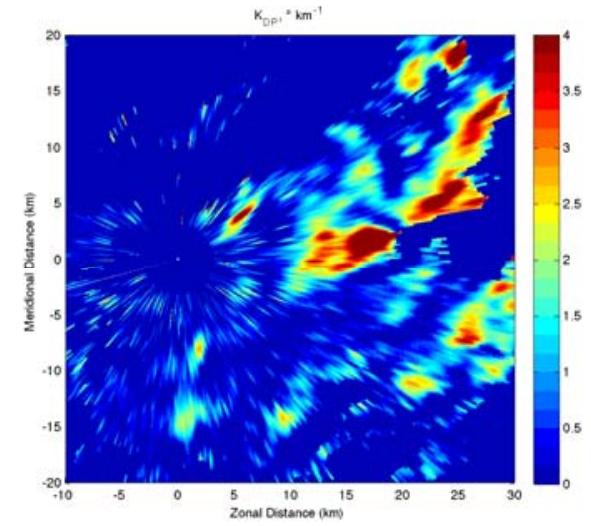

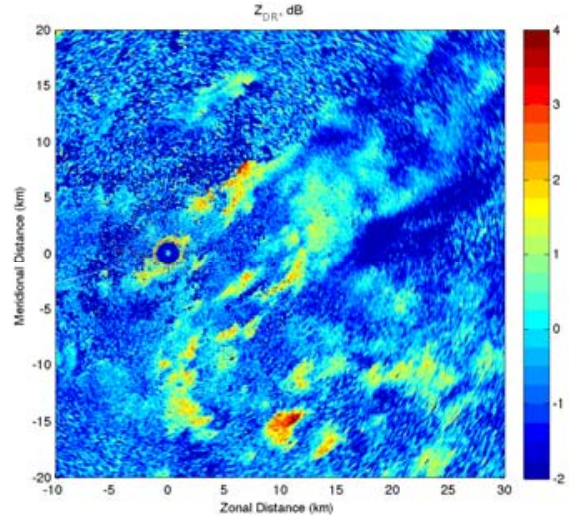

(d)

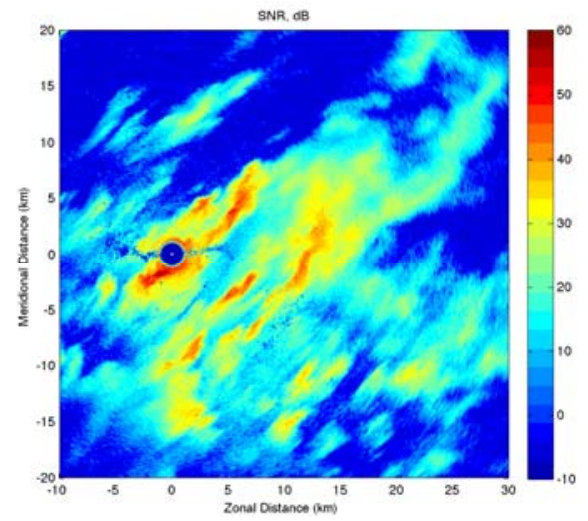

Fig. 10. X-band CASA IP1 KSAO radar observations: a) radar reflectivity; b) differential reflectivity; c) specific differential phase; and d) SNR (2 degree elevation angle, 1950 UTC on 24 April 2011). 

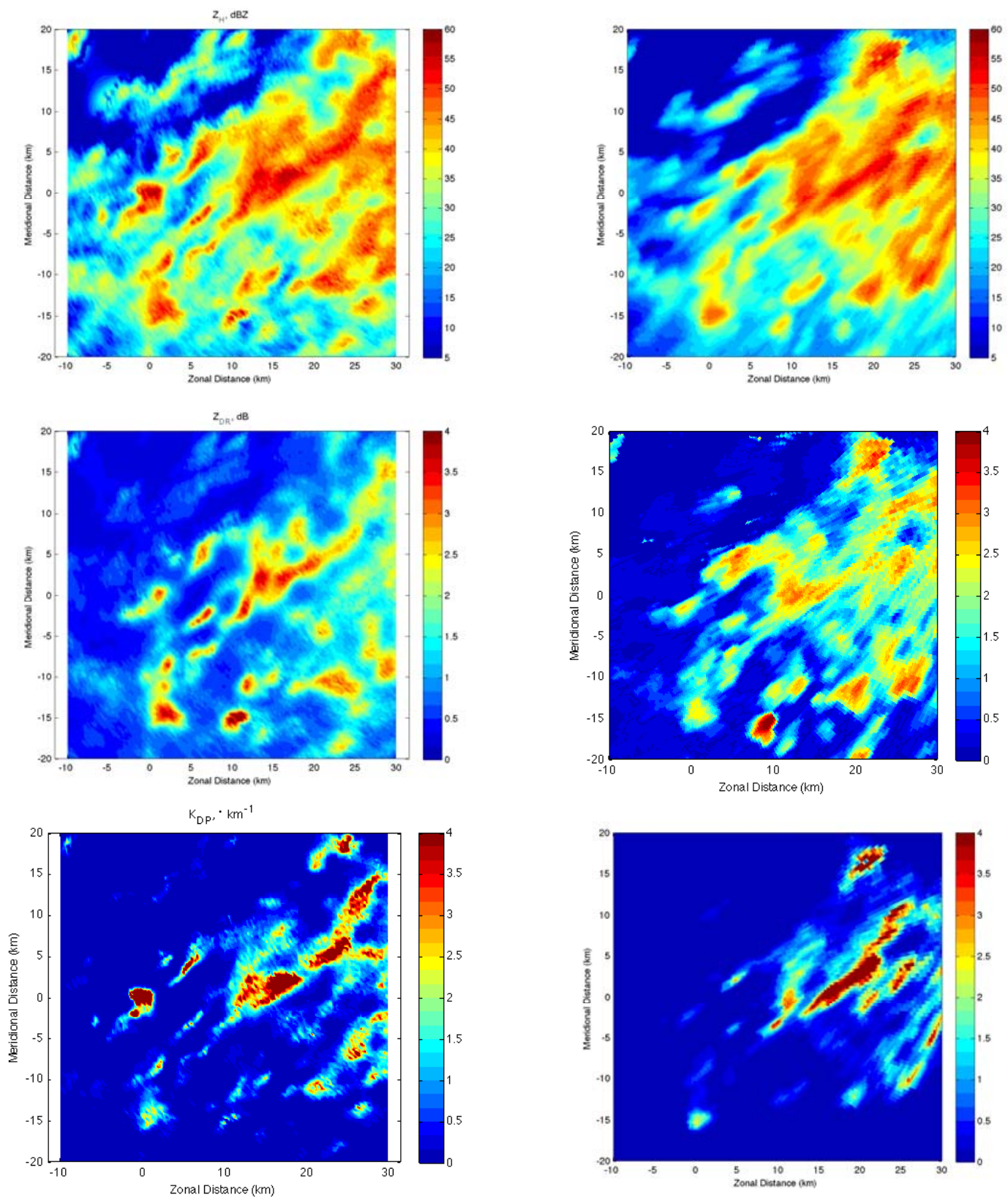

Fig. 11. Comparison of retrieval results (left column) and simulation results from S-band KOUN data (right column). The retrieval is based on X-band KSAO data shown in Fig. 10. Three rows from top to bottom show radar reflectivity, differential reflectivity, and specific differential phase, respectively. 